OPEN ACCESS

Edited by:

Janila Pasupuleti,

International Crops Research Institute

for the Semi Arid Tropics, India

Reviewed by:

Vikas Kumar Singh,

International Crops Research Institute for the Semi Arid Tropic, India

Radhakrishnan T,

ICAR-Directorate of Groundnut

Research, India

*Correspondence: Yarmilla Reinprecht

yreinpre@uoguelph.ca

Specialty section:

This article was submitted to Plant Genetics and Genomics,

a section of the journal

Frontiers in Genetics

Received: 31 October 2015

Accepted: 07 March 2016

Published: 29 March 2016

Citation:

Reinprecht $Y$ and Pauls KP (2016)

Microsomal Omega-3 Fatty Acid

Desaturase Genes in Low Linolenic

Acid Soybean Line RG10 and Validation of Major Linolenic Acid QTL.

Front. Genet. 7:38.

doi: 10.3389/fgene.2016.00038

\section{Microsomal Omega-3 Fatty Acid Desaturase Genes in Low Linolenic Acid Soybean Line RG10 and Validation of Major Linolenic Acid QTL}

\author{
Yarmilla Reinprecht ${ }^{*}$ and K. Peter Pauls \\ Department of Plant Agriculture, University of Guelph, Guelph, ON, Canada
}

High levels of linolenic acid $\left(80 \mathrm{~g} \mathrm{~kg}^{-1}\right)$ are associated with the development of off-flavors and poor stability in soybean oil. The development of low linolenic acid lines such as RG10 $\left(20 \mathrm{~g} \mathrm{~kg}^{-1}\right.$ linolenic acid) can reduce these problems. The level of linolenic acid in seed oil is determined by the activities of microsomal omega-3 fatty acid desaturases (FAD3). A major linolenic acid QTL (>70\% of variation) on linkage group B2 (chromosome Gm14) was previously detected in a recombinant inbred line population from the RG10 × OX948 cross. The objectives of this study were to validate the major linolenic acid QTL in an independent population and characterize all the soybean FAD3 genes. Four FAD3 genes were sequenced and localized in RG10 and OX948 and compared to the genes in the reference Williams 82 genome. The FAD3A gene sequences mapped to the locus Glyma.14g194300 [on the chromosome Gm14 (B2)], which is syntenic to the FAD3B gene (locus Glyma.02g227200) on the chromosome Gm02 (D1b). The location of the FAD3A gene is the same as was previously determined for the fan allele, that conditions low linolenic acid content and several linolenic acid QTL, including Linolen 3-3, mapped previously with the RG10 $\times$ OX948 population and confirmed in the PI 361088B $\times$ OX948 population as Linolen-PO (FAD3A). The FAD3B gene-based marker, developed previously, was mapped to the chromosome Gm02 (D1b) in a region containing a newly detected linolenic acid QTL [Linolen-RO(FAD3B)] in the RG10 $\times$ OX948 genetic map and corresponds well with the in silico position of the FAD3B gene sequences. $F A D 3 C$ and $F A D 3 D$ gene sequences, mapped to syntenic regions on chromosomes Gm18 (locus Glyma.18g062000) and Gm11 (locus Glyma.11g227200), respectively. Association of linolenic acid QTL with the desaturase genes FAD $3 A$ and $F A D 3 B$, their validation in an independent population, and development of FAD3 gene-specific markers should simplify and accelerate breeding for low linolenic acid soybean cultivars.

Keywords: soybean, $\omega-3$ fatty acid desaturase, FAD3 genes, QTL validation, gene-based markers, in silico, synteny 


\section{INTRODUCTION}

Soybean is grown primarily for high content of oil $\left(20 \mathrm{~g} \mathrm{~kg}^{-1}\right)$ and protein $\left(40 \mathrm{~g} \mathrm{~kg}^{-1}\right)$ in seed. Oil extracted from standard soybean cultivars contains approximately $110 \mathrm{~g} \mathrm{~kg}^{-1}$ palmitic acid (16:0), $50 \mathrm{~g} \mathrm{~kg}^{-1}$ stearic acid (18:0), $210 \mathrm{~g} \mathrm{~kg}^{-1}$ oleic acid (18:1), $550 \mathrm{~g} \mathrm{~kg}^{-1}$ linoleic acid (18:2), and $80 \mathrm{~g} \mathrm{~kg}^{-1}$ linolenic acid (18:3) (Wilcox, 1989). Although a source of omega fatty acid from plants, the normal level of linolenic acid in standard cultivars is associated with instability and short shelf life of soybean oil. Oxidation of linolenic acid results in the accumulation of undesirable odors and flavors in the oil, which reduces its palatability and food quality (Wilson et al., 1981; Kinney, 1997; Reinprecht et al., 2011). Soybean oil stability problems can be reduced by developing cultivars with low linolenic acid levels (Hammond and Fehr, 1975). For good flavor stability and increased storage life, the linolenic acid content of soybean seed should be $10 \mathrm{~g} \mathrm{~kg}^{-1}$ or less (Khanna and Singh, 1991). Oil extracted from low linolenic acid lines is more stable, needs less hydrogenation and contains lower levels of trans fatty acids than oil extracted from conventional soybean cultivars (Mounts et al., 1986; Clemente and Cahoon, 2009).

Fatty acid biosynthesis in plants is under developmental and tissue-specific control (Guerra and Holbrook, 1988). De novo fatty acid biosynthesis occurs in the chloroplast stroma of leaves and proplastids of seeds and their incorporation into triacylglycerides (TAGs) takes place in the endoplasmic reticulum (ER; Browse and Somerville, 1991; Ohlrogge et al., 1993). In higher plants, polyunsaturated fatty acids (PUFA) are synthesized through both prokaryotic and eukaryotic pathways (Browse et al., 1986). The two pathways are encoded by separate sets of genes (Harwood, 1988; Somerville and Browse, 1991).

In soybean seed, linolenic acid is synthesized by consecutive desaturation [the creation of a double bond (at the delta position) by the enzymatic removal of hydrogen from a methylene group in an acyl chain (Shanklin and Cahoon, 1998)] of oleic acid catalyzed by fatty acid desaturases (FADs, Cherif et al., 1975). Thus, two sets of genes might control linolenic acid content, which may act in additive, possibly sequential ways. The FAD2 genes encode omega ( $\omega)-6$ FADs and control the conversion of oleic acid into linoleic acid. The FAD3 gene family encodes $\omega-3$ FADs, which catalyzes transformation of linoleic acid into linolenic acid (Yadav et al., 1993).

The FADs possess common structural features and likely share a catalytic mechanism. Almost all membrane-bound FADs are characterized by the presence of a di-iron cofactor that interacts with three regions of conserved histidine motifs $\left(\mathbf{H X}_{(3-4)} \mathbf{H X}_{(7-41)} \mathbf{H X}_{(2-3)} \mathbf{H H X}_{(61-189)} \mathbf{H X}_{(2-3)} \mathbf{H H}\right)$, which are essential for catalytic activity (Shanklin et al., 1994). In addition, the amino acid sequence FVLGHDCGHGSF (which includes histidine box 1) is found in all known plant omega ( $\omega)-3$ FADs (Yadav et al., 1993).

The level of linolenic acid in soybean seed is determined by three FAD3 genes (FAD3A, FAD3B, and FAD3C; Bilyeu et al., 2003). At least three major loci (Fan, Fan2, and Fan3) and potentially a fourth (Fanx), have been identified with multiple alleles, including some with the reduced levels of linolenic acid (Rajcan et al., 2005; Lee et al., 2007). The Fan locus was mapped between the RFLP markers B124_1 and B194_1 on the linkage group (LG) B2 or chromosome Gm14 (Brummer et al., 1995). The first FAD3 gene was mapped to chromosome Gm14 (or linkage group B2, Byrum et al., 1995). A reduced level of linolenic acid in A5 was associated with full or partial deletion of a microsomal omega-3 desaturase gene (Byrum et al., 1995).

Loci associated with the low levels of linolnic acid in soybean have been linked to the genes encoding FAD3 desaturases and the annotated genome sequences (Bilyeu et al., 2011). FAD3A (FAD3-1b) gene corresponds to the Fan locus and, in the current Wm82.a2.v1 annotation of soybean genome sequence (Phytozome, http://phytozome. jgi.doe.gov/pz/portal.html; Goodstein et al., 2012), is represented as Glyma.14g194300 on the chromosome G14 (B2) (Glyma.14g194300/FAD3A/Fan). FAD3B (FAD3-1a) gene is analogous to the Fan3 locus and is represented as Glyma.02g227200 on the chromosome Gm02 (D1b) (Glyma.02g227200/FAD3B/Fan3). FAD3C (FAD3-2a) gene is the Fan2 locus and is represented as Glyma.18g062000 on the chromosome Gm18 (G) (Glyma.18g062000/FAD3C/Fan2). FAD3D (FAD3-2b) gene is at the locus Glyma.11g174100 on the chromosome Gm11 (B1). However, more work is needed to assign Glyma.11g174100/FAD3D (FAD3-2b) to any locus associated with the linolenic acid levels.

The FAD3 gene sequences were determined and deposited in GenBank (Bilyeu et al., 2003; Anai et al., 2005). They have similar structures and contain eight exons (Iba et al., 1993; Chi et al., 2011). The recent release of complete draft of the soybean genome sequence gives new insights into complex organization of this ancient paleopolyploid (Schmutz et al., 2010). Four regions containing FAD3 genes were identified in soybean genome (Chi et al., 2011; Gilman and Bilyeu, 2012).

The genetic basis of low linolenic acid soybean was associated with mutations in one or more FAD3 genes (Bilyeu et al., 2003, 2005, 2006; Anai et al., 2005; Chappell and Bilyeu, 2006, 2007; Reinprecht et al., 2009). For example, PI 361088B is a naturally-occurring mutant line which contains over $4 \%$ seed linolenic acid (Rennie et al., 1988). A two nucleotide insertion in the coding sequence of $F A D 3 A$ gene resulted in a frameshift and a premature stop codon (Chappell and Bilyeu, 2007). The $3 \%$ of linolenic acid content in the seed oil of the line 10-73 (derived from CX1512- 44) was associated with mutations in FAD3A and FAD3C genes (Bilyeu et al., 2005). Low linolenic acid line RG10, also contains less than $3 \%$ linolenic acid. The line was developed by remutagenesis (Stojšin et al., 1998) of the $4 \%$ linolenic acid line C1640 (Wilcox and Cavins, 1985). In addition to the FAD3A mutation from C1640 (Chappell and Bilyeu, 2006), RG10 contains a novel null allele of the FAD3B gene (Reinprecht et al., 2009). By combining mutations in all three $F A D 3$ genes from an RG10-derived soybean line (RCAT 0716L, GmFAD3aabbCC) and 10-73 (GmFAD3aaBBcc), Bilyeu et al. (2011) were able to produce novel soybean lines $(G m F A D 3 a a b b c c)$ containing only $1 \%$ linolenic acid in the seed oil. 
The current reference soybean (GmComposite_2003) genetic map [created using the 1999 ISU/USDA (Iowa State University/United States Department of Agriculture) map as the foundation] covers $2657.93 \mathrm{cM}$ of Kosambi map distance and contains 6394 markers and 3027 QTL on 20 linkage groups (LGs) or chromosomes [SoyBase (http://soybase.org/; accessed 26 Sep 2015); Grant et al., 2010]. Forty four QTL for linolenic acid were identified on 14 LGs (chromosomes) of the GmComposite_2003 map by 10 research groups.

We developed a molecular linkage map with 118 markers and identified QTL for linolenic acid content and seed lipoxygenases based on the RG10 × OX948 recombinant inbred line (RIL) population. Based on composite interval mapping (CIM), a major linolenic acid QTL (Linolen 3-3) that accounted for 72-78\% of linolenic acid variability, was positioned on LG B2 (Gm14, flanked by SSR marker Satt534 and FAD3 gene-specific marker FAD3i6; Reinprecht et al., 2006; SoyBase). Although strongly expressed in all three Ontario locations (Harrow, Ridgetown, and Woodslee in 2000) this QTL needed to be validated in different genetic backgrounds in order to be useful for marker-assisted selection (MAS) of soybean lines with reduced levels of linolenic acid. QTL regions are generally large and can contain thousands of potential candidate genes for the trait. The most robust marker for a QTL is located in the gene that determines the phenotype. In our previous work, $F A D 3 A$ gene-based markers were mapped to the region of the major linolenic acid QTL on the chromosome Gm14 (B2) in the RG10 × OX948 population (Reinprecht et al., 2009).

The objectives of the current study were to characterize all of the FAD3 genes in low linolenic acid genotype RG10 and a wild-type linolenic acid line (OX948) and to validate the major linolenic acid QTL (Linolen 3-3) in an independent PI 361088B $\times$ OX948 population. In a previous study (Reinprecht et al., 2009), conducted to determine molecular basis of low linolenic acid trait in RG10, we developed FAD $3 A$ and $F A D 3 B$ gene mutation-based markers. In this work, we developed markers for the other two FAD3 genes (FAD3C and FAD3D). The development of FAD3 gene-based markers provides simple, genotype-based selection assays that can be used in early stages of breeding for soybean with low levels of linolenic acid.

\section{MATERIALS AND METHODS}

\section{Plant Material}

Reciprocal crosses were made between the low linolenic acid mutant lines (RG10 and PI 361088B) and OX948 (seed lipoxygenase triple null line). PI $361088 \mathrm{~B}$ is a naturally-occurring low linolenic acid source (Rennie et al., 1988) and line RG10 was developed at the University of Guelph, Ridgetown Campus (Stojšin et al., 1998) by treating the low linolenic acid line C1640 with ethyl methanesulfonate (EMS). The allele at the Fan locus in RG10 is designated as fan-b, for a very low level of linolenic acid $\left(<25 \mathrm{~g} \mathrm{~kg}^{-1}\right)$. In line PI $361088 \mathrm{~B}$ the Fan allele is designated as fan (PI 361088B) for low level of linolenic acid $\left(\sim 39 \mathrm{~g} \mathrm{~kg}^{-1}\right)$ (Rennie et al., 1988). OX948 is a selection from a backcross between Harovinton and a triple lipoxygenase null (3lx) $F_{2}$ plant, and has high linolenic acid content $\left(>80 \mathrm{~g} \mathrm{~kg}^{-1}\right)$. The $3 \mathrm{~lx}$ genotype, produced by gamma irradiation, was obtained from Dr. Kitamura (National Agricultural Research Centre, Ministry of Agriculture, Forestry and Fisheries, Tsukuba, Japan).

Detailed descriptions of the development of the RIL populations were presented in Reinprecht et al. (2005). The RG10 × OX948 mapping population of $169 \mathrm{~F}_{5}$-derived RILs was used to develop a linkage map and identify QTL for a number of seed and agronomic traits, including the content of linolenic acid (Reinprecht et al., 2006). Single seed descent was used to advance the $\mathrm{F}_{3}$ seed to the $\mathrm{F}_{4}$ generation in Belize (Continuous Crop Improvement Co., Ltd., Belize City), where harsh conditions (hurricane) resulted in a significant reduction in the number of lines in the PI $361088 \mathrm{~B} \times$ OX948 confirmation population; from $300 \mathrm{~F}_{2}$ lines to $45 \mathrm{~F}_{5}$-derived RILs.

\section{Determination of Linolenic Acid Content and Activity of $\omega-3$ FAD}

Fatty acid composition was determined using a half-seed technique (Wilcox and Cavins, 1985). Approximately one third of the cotyledon tissue distal from the embryonic axis was used for fatty acid analyses and the rest of the seed containing the embryo was planted for the next generation. The fatty acid compositions of the seed samples (obtained by bulking 10 halfseeds per parental lines and RILs) were determined by a gas liquid chromatography of fatty acid methyl esters $\left(\mathrm{g} \mathrm{kg}^{-1}\right)$ according to the modified method of Bannon et al. (1987) as described in Reinprecht et al. (2005). The estimates of $\omega$-3 FAD activity (\%) were derived from measurements of linoleic and linolenic acids levels (Cherif et al., 1975) as follows: 18:2 ( $\omega-3)$ Desaturation $=$ $[(18: 3) /(18: 2+18: 3)] \times 100$. The content of fatty acids and the level of $\omega-3$ desaturation were analyzed using Proc Mix protocol in SAS Institute Inc (2009).

\section{Sequencing FAD3 Genes in RG10 and OX948}

PCR amplification and sequencing of the FAD3 genes are described in Reinprecht et al. (2009). Briefly, sequences for soybean seed FAD3 genes, available in public databases at the initiation of this study [G. max microsomal $\omega$-3 FAD mRNAs for FAD3A (GenBank accession AY204710), FAD3B (GenBank accession AY204711), FAD3C (GenBank accession AY204712), reported by Bilyeu et al. (2003) and FAD3D (FAD3-2b) (GenBank accession AB188198, Anai et al., 2005)], were aligned with the Arabidopsis thaliana microsomal FAD3 gene (GenBank accession D26508) sequence (Nishiuchi et al., 1994) using the multiple sequence alignment program ClustalW (Chenna et al., 2003), at the European Bioinformatic Institute (EBI, http://www.ebi.ac.uk/clustalw). Based on differences among the alignments, primers to specifically amplify six to nine overlapping fragments from each of the four FAD3 genes were designed [using Primer3 (Rozen and Skaletsky, 2000) and synthesized by Sigma-Aldrich Canada (Oakville, ON, Canada)]. In addition, the $1 \mathrm{~kb}$ flanking sequences of $F A D 3 A$ and $F A D 3 B$, and $2 \mathrm{~kb}$ of $F A D 3 C$ and $F A D 3 D$ were used to amplify the $5^{\prime}$ and $3^{\prime}$ untranslated regions (UTR) in FAD3 genes. The criteria for primer design included a length of 18 to 34 base pairs (bp), a GC 
content of 40 to $60 \%$, fewer than four contiguous identical bases, and a melting temperature between 55 and $65^{\circ} \mathrm{C}$. The FAD3 gene-specific primers used to amplify overlapping fragments for sequencing are listed in a Table S1.

The gene-based primers were used in PCR reactions with genomic DNA from RG10 (low linolenic acid line) and OX948 (wild-type line) to amplify FAD3 genes (FAD3A6027 bp, region Gm14:45,934,715..45,940,741; FAD3B-6082 bp, region Gm02:41,418,800..41,424,881; FAD3C-6887 bp, region $\mathrm{Gm} 18: 5,644,513 . .5,651,399$; and $F A D 3 D-7372 \mathrm{bp}$, region Gm11:19,007,492..19,014,883 in the current soybean Wm82.a2.v1 genome annotation). DNA was isolated from the young, freeze-dried leaf tissue in a $3 \%$ CTAB solution using the method of Doyle and Doyle (1990). PCR reactions were performed in a total volume of $20 \mu \mathrm{l}$ containing $1 \times$ PCR buffer (supplied with enzyme), $3 \mathrm{mM} \mathrm{MgCl} 2$ (supplied with enzyme), $0.1 \mathrm{mM}$ each of dNTPs (GE Healthcare Bio-Sciences Corp., Piscataway, NJ), 1.6 U Taq DNA Polymerase (Invitrogen Canada Inc., Burlington, ON, Canada), $5 \mu \mathrm{M}$ each of the forward and reverse primer and $25 \mathrm{ng}$ of soybean genomic DNA. The reaction mixture was amplified with a PTC- $100^{\mathrm{TM}}$ Programmable Thermal Controller (MJ Research, Inc., Watertown, MA, USA) using the program that consisted of an initial 2 min denaturation step at $94^{\circ} \mathrm{C}$, followed by 35 cycles of denaturation at $94^{\circ} \mathrm{C}$ for $30 \mathrm{~s}$, annealing at $55-65^{\circ} \mathrm{C}$ for $45 \mathrm{~s}$ and extension at $72^{\circ} \mathrm{C}$ for $1 \mathrm{~min}$, with a final extension at $72^{\circ} \mathrm{C}$ for $10 \mathrm{~min}$. The PCR products were run on a $1 \%(\mathrm{w} / \mathrm{v})$ agarose gel containing ethidium bromide in $1 \times \mathrm{TBE}$ buffer for $2 \mathrm{~h}$ at $100 \mathrm{~V}$ and visualized under UV light.

RG10 and OX948 PCR fragments were gel-purified and cloned (TOPO cloning kit, Invitrogen, according to the manufacturer's instructions) or used directly as template for cycle sequencing $\left(\mathrm{CEQ}^{\mathrm{TM}} 8000\right.$ genetic analysis system; Beckman Coulter Inc., Fullerton, CA, USA). After verification [BLAST searches at the National Center for Biotechnology Information (NCBI, http://www.ncbi.nlm.nih.gov/BLAST/) and Phytozome (http:// phytozome.jgi.doe.gov/pz/portal.html\#!search?show=BLAST) ] , sequences were assembled with the CAP3 program (Xiaoqiu and Madan, 1999). The exon/intron structure, initially determined by aligning genomic sequences with the available cDNA sequences, was confirmed with the reference sequences (NCBI and Phytozome). The gene structure was also analyzed with the FGenesh 2.6 (http://linux1.softberry.com; Salamov and Solovyev, 2000). Translation of nucleotide sequences was generated on ExPASy [Swiss Institute of Bioinformatics (SIB) Bioinformatics Resource Portal], http://www.expasy.ch/tools/dna.html). Verified RG10 and OX948 sequences of the FAD3A, FAD3B, $F A D 3 C$, and $F A D 3 D$ genes were submitted to GenBank under accession numbers KU310958-KU310965.

\section{Development of Molecular Markers for FAD $3 C$ and FAD3D Genes}

Polymorphisms among FAD3 genes from different sources were determined by comparing the mutant RG10 with a wildtype OX948 sequences and the wild-type Williams82 (Wm82) reference sequences. Sequence differences between RG10 and
OX948 were used to design primers, develop molecular markers, and map FAD3C and FAD3D genes.

Because of the high similarity, FAD $3 C$ and FAD $3 D$ gene sequences were aligned and unique PCR primers were designed for both genes (FAD3C gene: forward $5^{\prime}$-TGA ATAAGCTGGCTTAGAAGTCAA- $3^{\prime}$ and reverse $5^{\prime}$-CTTTCA AAGATTTATTTACTACATTCTAAA- $3^{\prime}$; FAD3D gene: forward $5^{\prime}$-CCGGCGAGTGTCTTATGAACG-3' and reverse $5^{\prime}$-CACT TGGTATCCCAACCTTCGAG-3'). The PCR mixture, PCR program (except for the annealing step, which was performed at $63^{\circ} \mathrm{C}$ for the FAD3C SNP and at $60^{\circ} \mathrm{C}$ for the FAD3D marker) and electrophoresis conditions were same as for the FAD3 genes sequencing. Polymorphic primers were screened with the $169 \mathrm{~F}_{5}$ RILs from the mapping RG10 $\times$ OX948 population and 45 RILs from the verifying PI $361088 \mathrm{~B} \times \mathrm{OX} 948$ population.

\section{FAD3 Genes Mapping}

\section{In silico Mapping and Synteny Analysis}

The RG10 and OX948 FAD3 gene sequences were BLASTed against the soybean Wm82.a2.v1 genome sequence (Phytozome). They mapped to four chromosomes (Gm02, Gm11, Gm14, and Gm18) and the sequence coordinates [the beginning and end positions (in bp) on the chromosome] were retrieved. The start positions for each gene were used to map (in silico) RG10 and OX948 FAD3 gene sequences on the soybean sequence map. In addition, genome sequence (Wm82.a2.v1) coordinates for the Consensus map 4.0 markers on the chromosomes Gm14, Gm02, $\mathrm{Gm} 18$, and Gm11 were retrieved from SoyBase. Coordinates for the missing markers were obtained by BLASTing their sequences (indicated by "s" in marker name; SoyBase) against the soybean genome (Phytozome). Genetic maps (GmComposite_2003) coordinates for all features on these four chromosomes were downloaded from SoyBase. In this study, only QTL for linolenic acid were used.

Syntenic regions containing FAD3 genes in soybean genome were determined using locus identifier (Glyma.14g194300, Glyma.02g227200, Glyma.18g062000, and Glyma.11g174100) in PGDD (Plant Genome Duplication Database, http://chibba. agtec.uga.edu/duplication/index/locus; Lee et al., 2013) against complete genome sequences available for 47 flowering plant species. Synteny analysis was also performed in CoGe's (Comparative Genomics) GEvo (Genome Evolution Analysis tool) using BlastZ alignment algorithm and a $40 \mathrm{Mb}$ window (https://genomevolution.org/CoGe/GEvo.pl; Lyons and Freeling, 2008; Lyons et al., 2008).

\section{Genotyping with SSRs}

To connect different populations and various maps, SSR markers in FAD3 gene regions on chromosomes Gm02 (D1b), Gm11 (B1), Gm14 (B2), and Gm18 (G) (based on in silico map data) were selected from the GmComposite_2003 map. Primers for the SSR markers were screened with parental genomic DNA (RG10, PI 361088B, and OX948) for polymorphisms in PCR reaction mixtures that were identical to those used for sequencing of FAD3 genes. The SSR amplification program consisted of an initial denaturation for $2 \mathrm{~min}$ at $94^{\circ} \mathrm{C}$, followed by 35 cycles with denaturation at $94^{\circ} \mathrm{C}$, annealing at $48^{\circ} \mathrm{C}$ and extension 
at $68^{\circ} \mathrm{C}$, each for $45 \mathrm{~s}$ with a final extension for $5 \mathrm{~min}$ at $72^{\circ} \mathrm{C}$. The PCR products were separated by electrophoresis on $3 \%(\mathrm{w} / \mathrm{v})$ super fine resolution agarose (Amresco, Solon, $\mathrm{OH}$, USA) containing ethidium bromide in a $1 \times \mathrm{TBE}$ buffer at $100 \mathrm{~V}$ for $3 \mathrm{~h}$ and visualized under UV light. Polymorphic markers were used with DNA samples from 169 RILs from the RG10 × OX948 mapping population and 45 RILs from a validating population obtained from the PI $361088 \mathrm{~B} \times$ OX948 cross.

\section{Linkage Analysis}

The new SSR and FAD3 gene-specific markers were added to the regions containing the FAD3 genes in the existing RG10 $\times$ OX948 map (Reinprecht et al., 2006) and used to develop a linkage map for the validating PI $361088 \mathrm{~B} \times$ OX948 population. Linkage analysis was performed using Mapmaker/EXP version 3.0b (Lander et al., 1987) with a minimum LOD score of 3.0 and maximum distance between two markers of $50.0 \mathrm{cM}$. Recombination frequencies were converted to $\mathrm{cM}$ distances using Kosambi’s mapping function (Kosambi, 1944).

\section{QTL Analysis}

QTL analysis was performed with composite interval mapping (CIM). The QTL number, their chromosomal locations and allelic effects were determined by CIM using QTL Cartographer version 2.5 (Wang et al., 2011) with forward and backward regression model (map function Kosambi, window size of $10.0 \mathrm{cM}$, five control markers and speed walk of $2.0 \mathrm{cM})$. QTL were declared significant if their LOD threshold values were $\geq 2.0$. The location of a QTL was identified by the position of the peak in the significant LOD interval. The allelic effects at each significant QTL and the percentages of the phenotypic variation $\left(R^{2}\right)$ accounted by the identified QTL were obtained directly from the CIM output. Because the RIL populations were in the $F_{5}$ generation only additive effects were considered. The locations and effects of the linolenic acid QTL identified in the current study were compared with fatty acid QTL previously mapped to the GmComposite_2003 map (SoyBase).

\section{Maps Alignments}

Using common SSRs as anchors, the RG10 $\times$ OX948 linkage map was compared with the PI $361088 \mathrm{~B} \times$ OX948 map, as well as the GmComposite_2003 (SoyBase) genetic and sequence maps (Phytozome). On the sequence map, markers associated with the linolenic acid QTL were indicated with the bracketed QTL name (SoyBase and/or original publication). Maps were drawn in MapChart (Voorrips, 2002). Sequence and genetic maps containing FAD3 (FAD3A, FAD3B, FAD3C, and FAD3D) genes were connected manually using shared (in general, classical SSR) markers. Naming of maps was based on soybean chromosome number to linkage group assignment (SoyBase).

\section{RESULTS}

\section{Linolenic Acid Content and $\omega-3$ FAD Activity in Parental Lines}

The parental lines RG10, PI 361088B, and OX948 had significantly different levels of unsaturated fatty acids and differed in their relative $\omega-3$ (18:2) desaturase activities (Table 1). In particular, RG10 contained $23 \mathrm{~g} \mathrm{~kg}^{-1}$, PI 361088B $54 \mathrm{~g} \mathrm{~kg}^{-1}$, and OX948 $128 \mathrm{~g} \mathrm{~kg}^{-1}$ linolenic acid. The mechanisms of fatty acid desaturation proposed for the parental lines and the differences in the rate of $\omega-3$ FAD activities are presented in Figure 1. Based on this model, the low levels of linolenic acid in RG10 and PI 361088B are results of low activities of the relative $\omega-3$ fatty acid desaturation (3.4 and 7.8\%, respectively) in seeds of these genotypes. The much higher level of linolenic acid in a wildtype line (OX948) is a result of $20.5 \%$ relative $\omega-3$ FAD activity in this line (Figure 1).

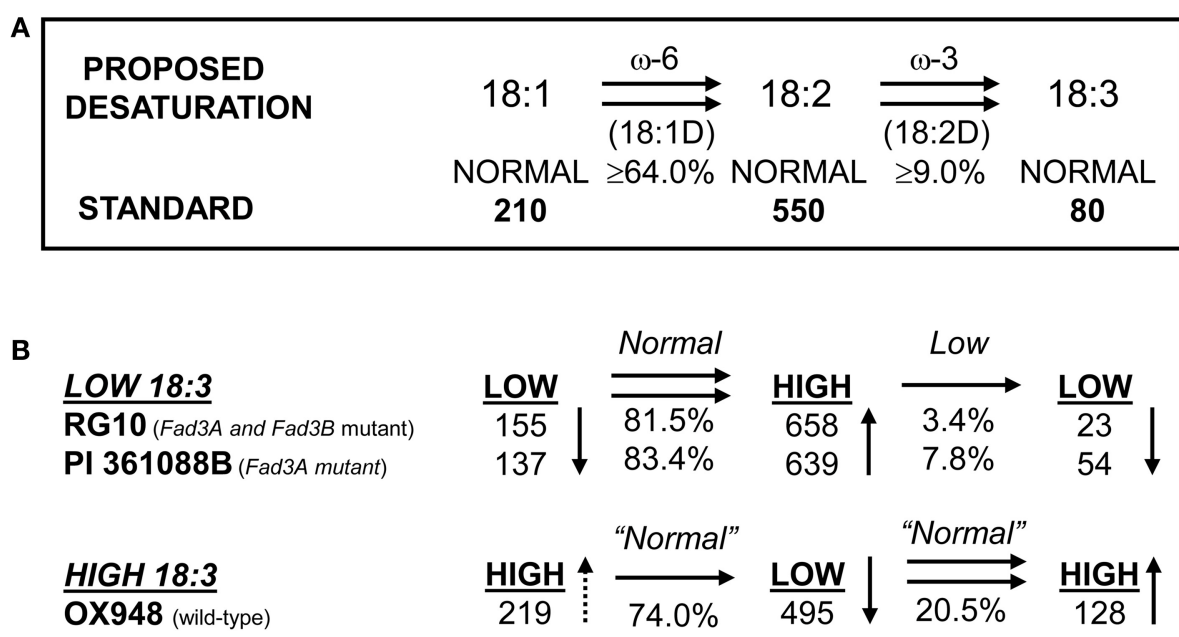

FIGURE 1 | Proposed mechanism of fatty acid desaturation in seeds of soybean lines RG10 and OX948. (A) Desaturation steps after Wilson et al. (1990); Wilson (1991); (B) Desaturation steps proposed for parental lines RG10 (EMS FAD3A and FAD3B genes mutant:), PI 361088B (naturally occurring FAD3A gene mutant), and OX948 (wild-type). 
TABLE 1 | Fatty acid composition and relative $\omega-3$ fatty acid desaturase activity of soybean parental lines RG10, PI 361088B, and OX948.

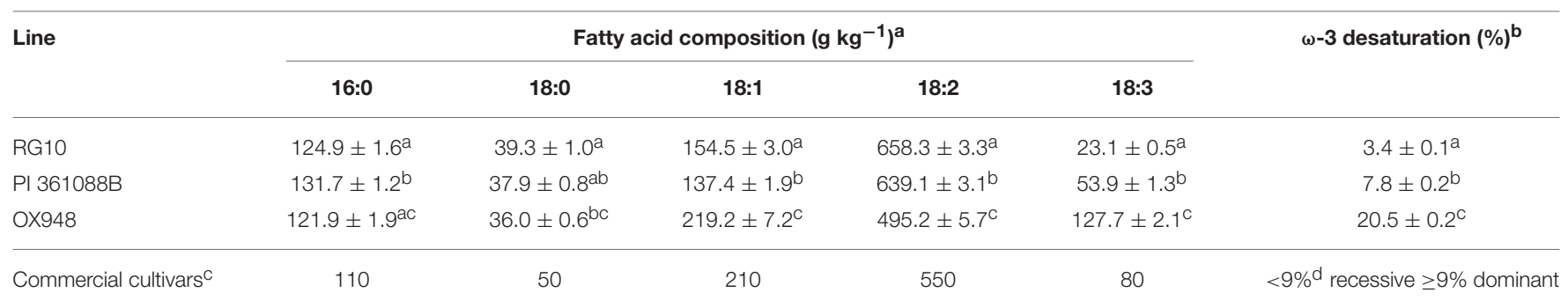

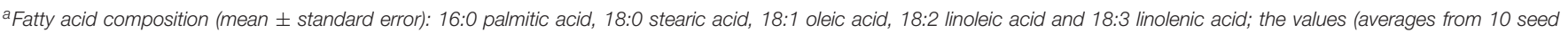
analyses) followed by the same letter are not significantly different at the 0.05 probability level based on the t-tests

${ }^{b}$ Desaturation: 18:2 D ( $\omega$-3 desaturase $)=[(18: 3) /(18: 2+18: 3)] \times 100$ (Cherif et al., 1975).

${ }^{c}$ (Wilcox, 1989).

${ }^{d}$ (Wilson et al., 1990).

\section{FAD3Genes in RG10 and OX948 and Comparison with FAD3 Genes in Reference Williams 82 (Wm82)}

Comparisons of the sequences for the four FAD3 genes from the low linolenic acid (RG10) and wild-type line (OX948) to the GenBank/Phytozome sequences (Figure S1-S4) confirmed that the amplified DNA fragments corresponded to FAD $3 A$, FAD 3B, FAD3C, and FAD3D (FAD3-2b, Anai et al., 2005) genes, respectively.

\section{FAD3A Gene}

The sizes of the FAD3A genes sequenced in this study were 5766 bp in RG10 and 5764 bp in OX948 including, approximately, $1000 \mathrm{bp}$ upstream and $860 \mathrm{bp}$ downstream of the open reading frame (ORF) (Table 2; Figure 2). The "TATA" box (TATAAA) is located between nucleotides 915 to 920 in RG10 and 913 through 918 in OX948. The start codon (ATG) occurs at position 1043 in RG10 and 1041 in OX948. The stop codon (TGA) is at 4909 in RG10 and 4907 in OX948. The polyadenylation [poly(A)] signal is located at position 5086 in RG10 and 5084 in OX948 (Figure S1). The OX948 ORF (1131 bp) encodes 376 amino acids with an estimated molecular weight of 43,945 daltons. Because of a mutation that introduces a stop codon in exon 6 (Reinprecht et al., 2009), the RG10 ORF (1131 bp) encodes a smaller protein of 265 amino acids with an estimated molecular weight of 30,783 daltons.

The comparison of the FAD3A genes in RG10 and OX948 with the corresponding Wm82 (current annotation Wm82.a2.v1) sequence revealed differences in both coding and noncoding regions (Figure 2). Two substitutions were detected in the coding region. One change in exon 2 (A to G) in OX948 did not change the amino acid (leucine, L109) that was encoded (CTA to CTG). The second change, detected in exon 6 in RG10, results in a substitution of an A for a G, and a codon change from TGG for tryptophan (W236) in OX948 and Wm82 to a stop codon (TGA) in RG10. Several differences among the sequences were found in intronic and UTR regions. A single nucleotide change (C to T) was detected in intron 1 of RG10 and OX948 compared to the $\mathrm{Wm} 82$ sequence. One $\mathrm{T}$ to $\mathrm{C}$ change was detected in intron 3 in RG10 and two T insertions were found in the OX948 sequences. One $\mathrm{T}$ and $3 \mathrm{~T}$ deletions were detected in intron 6 in RG10 and
OX948, respectively, compared to the reference Wm82 sequence. In addition, OX948 had 25 single nucleotide substitutions in the $5^{\prime}$ UTR sequence and two G to A changes and 28 Ns in its $3^{\prime}$ UTR region compared to the RG10 and Wm82 sequences (Figure 2 and Figure S1).

\section{FAD3B Gene}

The sizes of the $F A D 3 B$ gene sequenced in this study were 5984 bp in RG10 and 5985 bp in OX948 (Table 2; Figure 2). The "TATA" box occurs between nucleotides 808 and 813 in RG10 and between nucleotides 805 to 810 in OX948. The start codon (ATG) occurs at position 943 in RG10 and 940 in OX948. The stop codon (TGA) is located at 4849 in RG10 and 4850 in OX948. The poly (A) signal is in position 5009 in RG10 and 5010 in OX948 (Figure S2). The OX948 ORF (1143 bp) encodes 380 amino acids with an estimated molecular weight of 44,136 daltons. Because of the mutation at a splicing junction of intron 5 (Reinprecht et al., 2009), the RG10 ORF (1143 bp) encodes a smaller protein of 185 amino acids with an estimated molecular weight of 21,234 daltons.

A comparison of the FAD3B genes of the mutant (RG10) line and wild-type line (OX948) with the reference Wm82 sequence identified a single point mutation ( $\mathrm{T}$ to $\mathrm{C}$ ) in exon 5 of the sequence (Figure 2), which did not change the amino acid (phenylalanine, F204) encoded in this position in RG10 and OX948. Differences between OX948 and RG10 compared to the reference sequence were also detected in intronic regions including: in intron 1 (4T deletion in RG10 and $2 \mathrm{~T}$ insertion in OX948), intron 2 ( $\mathrm{C}$ to $\mathrm{T}$ and $\mathrm{A}$ to $\mathrm{T}$ changes in both RG10 and OX948), intron 5 ( $\mathrm{G}$ to A change in RG10 and T insertion in both RG10 and OX948), intron 6 [ $\mathrm{G}$ to A and $2 \mathrm{~T}$ insertions in RG10, G to T change in OX948 and several changes detected in both RG10 and OX948 (G to A substitution, C to G change, two single nucleotide insertions and one nine-nucleotide insertion)], and in intron 7 (four single nucleotide substitutions and one A insertion in both RG10 and OX948 compared to the reference). In the FAD3B $5^{\prime}$ UTR of the RG10 sequence a single $\mathrm{T}$ to $\mathrm{C}$ substitution was found. In the $5^{\prime} \mathrm{UTR}$ of the OX948 sequence, three substitutions ( $A$ to $\mathrm{G}, \mathrm{A}$ to $\mathrm{C}$, and $\mathrm{T}$ to $\mathrm{A}$ ) and a $\mathrm{C}$ deletion were found, compared to the RG10 and reference Wm82 sequences. Both RG10 and OX948 had a single G to 
TABLE 2 | Nucleotide sizes (base pairs, bp) of exons and introns and predicted proteins (amino acids, aa; dalton, Da) of four soybean $\omega-3$ fatty acid desaturase (FAD3) genes in RG10, OX948, and Williams 82 (Wm82).

\begin{tabular}{|c|c|c|c|c|c|c|c|c|c|c|c|c|c|}
\hline \multirow{2}{*}{\multicolumn{2}{|c|}{ Structure }} & \multicolumn{12}{|c|}{$\omega-3$ fatty acid desaturase (Fad3) gene ${ }^{a}$} \\
\hline & & RG10 & OX948 & Wm82 & RG10 & OX948 & Wm82 & RG10 & OX948 & Wm82 & RG10 & OX948 & Wm82 \\
\hline $5^{\prime}$ UTR & & 1042 & 1040 & 1090 & 942 & 939 & 1003 & 2066 & 2066 & 2083 & 2076 & 2078 & 2133 \\
\hline Exon 1 & & 293 & 293 & 293 & 305 & 305 & 305 & 296 & 296 & 296 & 299 & 299 & 299 \\
\hline Intron 2 & & 324 & 324 & 324 & 348 & 348 & 348 & 123 & 123 & 123 & 121 & 121 & 121 \\
\hline Exon 3 & & 67 & 67 & 67 & 67 & 67 & 67 & 67 & 67 & 67 & 67 & 67 & 67 \\
\hline Intron 3 & & 135 & 137 & 135 & 142 & 142 & 142 & 166 & 166 & 166 & 164 & 164 & 169 \\
\hline Exon 4 & & 93 & 93 & 93 & 93 & 93 & 93 & 93 & 93 & 93 & 93 & 93 & 93 \\
\hline Intron 4 & & 109 & 109 & 109 & 98 & 98 & 98 & 156 & 156 & 156 & 219 & 219 & 219 \\
\hline Exon 7 & & 138 & 138 & 138 & 138 & 138 & 138 & 138 & 138 & 138 & 138 & 138 & 138 \\
\hline Intron 7 & & 673 & 673 & 673 & 627 & 627 & 626 & 277 & 277 & 277 & 323 & 323 & 323 \\
\hline Exon 8 & & 183 & 183 & 183 & 183 & 183 & 183 & 192 & 192 & 192 & 192 & 192 & 192 \\
\hline $3^{\prime}$ UTR & & 857 & 857 & 1069 & 1135 & 1135 & 1183 & 2226 & 2227 & 2270 & 1685 & 1083 & 2275 \\
\hline Total DNA & & 5766 & 5764 & 6027 & 5984 & 5985 & 6082 & 6826 & 6827 & 6887 & 6720 & 6120 & 7372 \\
\hline Total coding region & & 1131 & 1131 & 1131 & 1143 & 1143 & 1143 & 1143 & 1143 & 1143 & 1146 & 1146 & 1146 \\
\hline Total introns & & 2736 & 2736 & 2737 & 2764 & 2768 & 2753 & 1391 & 1391 & 1391 & 1813 & 1813 & 1818 \\
\hline TATA-box & & 915 & 913 & 963 & 808 & 805 & 869 & 1663 & 1663 & 1680 & 2009 & 2011 & 2066 \\
\hline Poly (A) & & 5086 & 5084 & 5135 & 5009 & 5010 & 5059 & 4961 & 4961 & 4978 & 5481 & 5483 & 5543 \\
\hline
\end{tabular}

a FAD3A: KU310962 (RG10), KU310958 (OX948), Glyma.14g194300 [Wm82 (Williams 82)]; FAD3B: KU310963 (RG10), KU310959 (OX948), Glyma.024g227200 [Wm82 (Williams 82)]; FAD3C: KU310964 (RG10), KU310960 (OX948), Glyma.18g062000 [Wm82 (Williams 82)]; FAD3D: KU310965 (RG10), KU310961 (OX948), Glyma.11g174100 [Wm82 (Williams 82)]. ${ }^{b}$ Predicted protein size (amino acid, aa) and molecular weight (dalton, Da; computed at ExPaSy at http://web.expasy.org/compute_pi).

${ }^{c}$ Mutations ("M) in FAD3A and FAD3B genes, respectively (Reinprecht et al., 2009).

A substitution at the $3^{\prime} \mathrm{UTR}$ of the FAD3B gene (Figure 2 and Figure S2).

\section{FAD3C Gene}

The sizes of the $F A D 3 C$ gene sequenced in the present study were 6826 bp in RG10 and 6827 bp in OX948 (Table 2; Figure 2). The "TATA" box is located between nucleotides 1663 through 1668 in both RG10 and OX948. The start codon (ATG) occurs at position 2067 in both RG10 and OX948. The stop codon (TAA) is at position 4600 in both RG10 and OX948. The poly (A) is located at position 4961 in both RG10 and OX948 (Figure S3). The ORF (1143 bp) encodes a protein of 380 amino acids with an estimated molecular weight of 43,937 daltons in both RG10 and OX948.

There were no differences in the coding or intronic regions of the FAD3C genes in RG10 and OX948 when compared to the $\mathrm{Wm} 82$ reference sequence. The only changes detected were two $\mathrm{A}$ to G substitutions in the $5^{\prime} \mathrm{UTR}$ and one T deletion in the $3^{\prime} \mathrm{UTR}$ in the RG10 sequence compared to the OX948 and the Wm82 reference sequences. In the $3^{\prime} \mathrm{UTR}$ of the OX948 sequence, a single A to G substitution was found, compared to the RG10 and reference Wm82 sequences (Figure 2 and Figure S3).

\section{FAD3D Gene}

The sizes of the $F A D 3 D$ gene sequenced in the present study were 6720 bp in RG10 and 6120 bp in OX948 (Table 2; Figure 2). The "TATA" box is located between nucleotides 2009 through 2014 in RG10 and from 2011 to 2016 in OX948. The start codon (ATG) occurs at position 2077 in RG10 and 2079 in OX948. The stop codon (TAA) is at 5035 in RG10 and 5037 in OX948. The poly (A) is located at position 5481 in RG10 and at 5483 in OX948 (Figure S4). The ORF (1146 bp) encodes a protein of 381 amino acids with an estimated molecular weight of 44,072 daltons in both RG10 and OX948.

Two single nucleotide differences were detected in the coding regions of both the RG10 and OX948 FAD3D genes compared to the Wm82 reference sequence (Figure 2). An A to $\mathrm{T}$ substitution in exon 2 changed methionine M110 to leucine 
A

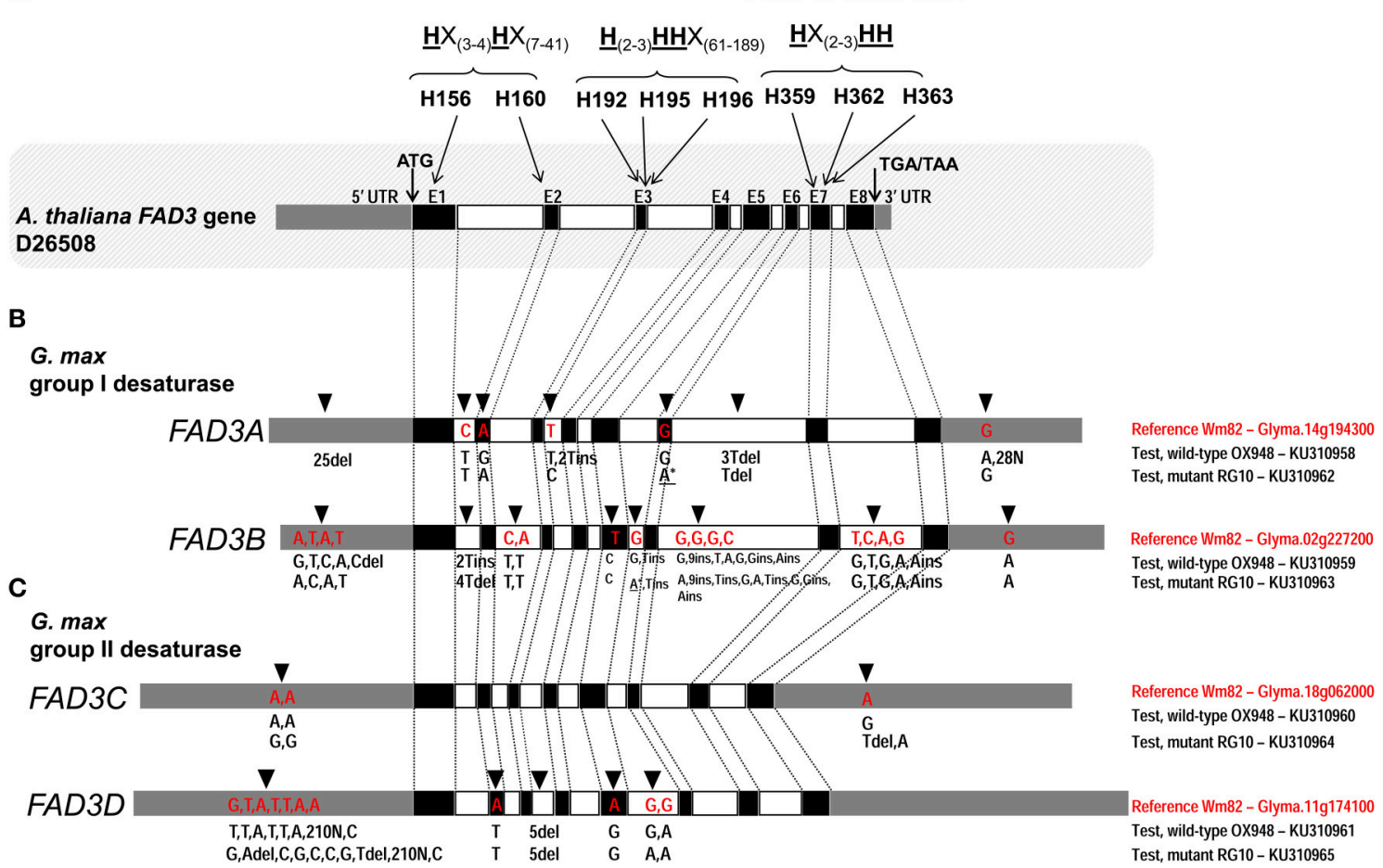

FIGURE 2 | Comparison of the $\omega-3$ fatty acid desaturase (FAD3) genes in test lines, RG10 and OX948, with the Williams 82 (Wm82) reference sequence. (A) Arabidopsis thaliana FAD3 gene; three conserved histidine boxes (exons 1-2, 3, and 7) and iron-binding sites are indicated. (B) Glycine max FAD3A and FAD3B genes; (C) Glycine max FAD3C and FAD3D genes. Germplasm, gene and GenBank accession are indicated for each sequence on the right of each gene model. In each bar, black boxes represents exons (E1-E8), clear boxes represent introns and gray boxes represent untranslated regions (UTR). The positions of the unique features in each sequence are indicated by an arrow [on the top of the bar (Wm82)] and the feature type (ATT/C/G for a single nucleotide difference and ins/del for inserion and deletions). The positions of mutations in FAD3A and FAD3B genes in RG10 are indicated by an asterisk (*).

and the substitution of an $\mathrm{A}$ for a $\mathrm{G}$ in exon 5 changed the codons for aspartate D232 to glycine in OX948 and RG10. Five nucleotide deletions in intron 3 were detected in RG10 and OX948 compared to the Wm82 reference sequence. One $\mathrm{G}$ to $\mathrm{A}$ substitution was detected in the intron 5 sequence of RG10 relative to Wm82. Additional G to A substitutions (relative to Wm82) were found in both RG10 and OX948. Further differences were found in the $5^{\prime} \mathrm{UTR}$ regions. Five single base substitutions and two deletions ( $\mathrm{A}$ and $\mathrm{T}$ ) were detected in the RG10 5'UTR when compared to the OX948 and the reference Wm82 sequences. A single $G$ to $T$ substitution was found in the OX948 sequence relative to the other two. Additional A to C substitutions were detected in both the RG10 and OX948 sequences compared to the Wm82. Because of the low quality sequencing, a sequence stretch of 210 bp (position 1524-1733 in Wm82) is missing from RG10 and OX948 $5^{\prime} \mathrm{UTR}$ regions of the $F A D 3 D$ gene (Figure 2 and Figure $S 4$ ).

The four FAD3 genes in RG10 and OX948 have similar structures, consisting of eight exons and seven introns (Table 2; Figure 2). While the sizes of exons two to seven are conserved among the four FAD3 genes, the size of exon 1 is different in all four genes (293 bp in FAD3A, 305 bp in FAD3B, 296 bp in $F A D 3 C$, and 299 bp in $F A D 3 D$ ). Exon 8 is slightly larger in $F A D 3 C$ and FAD $3 D$ genes (192 bp) compared to the FAD $3 A$ and
$F A D 3 B$ genes (183 bp). In contrast, intron 7 has a different size in the four FAD3 genes. Among the four genes, FAD3A has the shortest intron 3 and the longest intron 7; FAD $3 B$ gene has the longest introns 2 and 6 and the shortest introns 4 and 5; FAD3C has the longest intron 3 and the shortest introns 1 and 7; and $F A D 3 D$ is characterized by the longest introns 1,4 , and 5 and the shortest introns 2 and 6. FAD $3 C$ gene has the smallest total intron size (1391 bp). The FAD3A and FAD3B genes have similar total intron sizes (2736 bp and $2764 \mathrm{bp}$, respectively), which is almost double that found in FAD3C (Table 2). All introns start with GT (except intron 5 in RG10 FAD3B gene) and end with AG. The FAD3A ORF (OX948) encodes the smallest protein (376 amino acids in wild-type OX948) while the ORFs of other three genes encode slightly larger proteins of similar sizes (FAD3B, encodes 380 amino acids in wild-type OX948; FAD3C and FAD3D, encode 381 amino acids in OX948; Figure S5).

\section{Positions of the FAD3 Genes in Sequence and Genetic Maps and Correspondence to Linolenic Acid QTL}

In soybean genome, four FAD3 genes are positioned on four different chromosomes. Numerous syntenic regions were found for soybean $F A D 3$ genes with 47 plant species that are currently 
TABLE 3 | Syntenic blocks containing FAD3 loci in soybean (Wm82.a2.v1) genome.

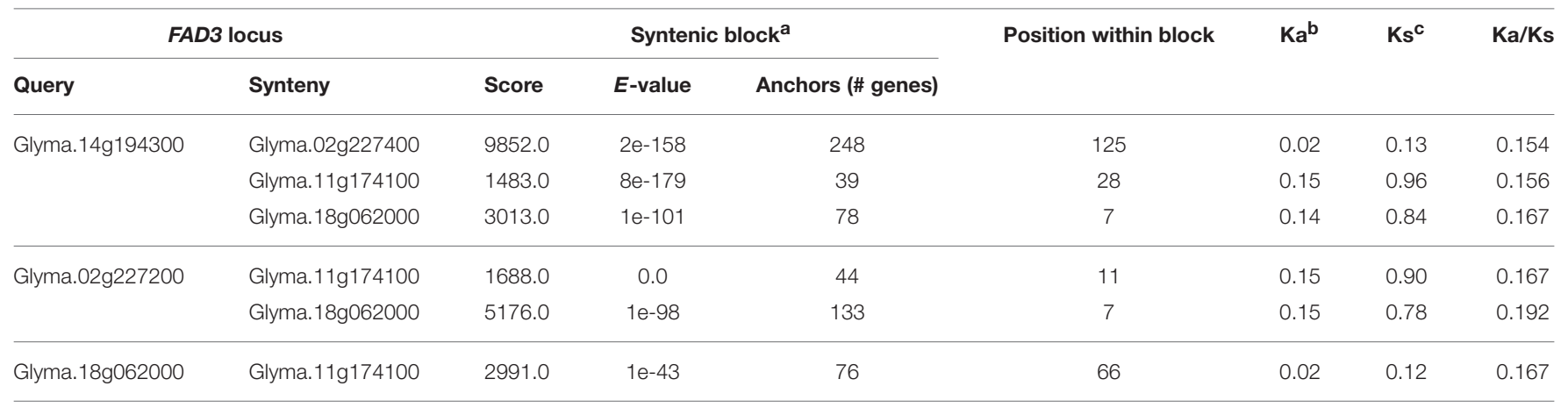

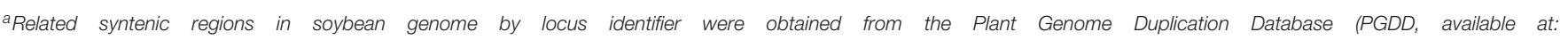
http://chibba.agtec.uga.edu/duplication/index/locus; accessed 2 Oct 2015).

${ }^{b}$ Nonsynonymous substitution rates.

cSynonymous substitution rates.

available in PGDD (Table S2). Soybean Glyma.14g194300 (FAD3A gene) on the chromosome Gm14 was syntenic to 52 regions in 27 different plant species (6 to 248 anchor genes). Forty nine syntenic blocks were found for Glyma.02g227200 (FAD2B gene) with 25 plant species (6 to 254 anchors) and 53 for Glyma.18g062000 (FAD3C gene) with 27 plant species (6 to 302 anchors). The smallest number of syntenic regions was found for Glyma.11g174100 (FAD3D gene). The region on the chromosome Gm11 containing Glyma.11g174100 was syntenic to 36 regions in 21 plant species ( 7 to 76 anchor genes).

Regions containing FAD3 genes in soybean genome are syntenic (Table 3). Group I $\omega-3$ FAD genes FAD $3 A$ and $F A D 3 B$ are found on homeologous regions of chromosomes Gm14 (B2) and Gm02 (D1b), respectively. Large duplication blocks consist of 4,551,566 bp in Gm14 (position Gm14:43,488,986..48,040,552) and 4,093,688 bp in Gm02 (position Gm02:39,317,568..43,411,256) with 248 anchor genes (Figures 3A, 4 and Table S2). The FAD3A gene is at the locus Gm14.g194300 on the chromosome Gm14 (position Gm14:45,935,668..45,939,896) and the FAD3B gene is at the locus Gm.02g227200 on the chromosome Gm02 (position Gm02:41,419,656..41,423,881; Wm82.a2.v1). Group II desaturase genes, FAD $3 C$ and $F A D 3 D$, are found on homeologous regions on the chromosomes Gm18 (G) and Gm11 (D1b), respectively. Duplication blocks consist of 1,160,704 bp (position Gm18:4,627,923..5,788,627) in Gm18 and 9,800,783 bp in Gm11 (position Gm11:14,448,495..24,249,278) with 76 anchor genes (Figures 3B, 4 and Table S2). The FAD3C gene is at the locus Gm.18g062000 on the chromosome Gm18 (position $\mathrm{Gm} 18: 5,646,502 . .5,649,337$ ) and the $F A D 3 D$ gene is at the locus Gm.11g174100 on the chromosome Gm11 (position Gm11:19,009,581..19,012,951).

\section{FAD3A Gene, Fan Allele, and Linolenic Acid QTL}

BLAST searches against the soybean genome (current annotation Wm82.a2.v1; http://phytozome.jgi.doe.gov/pz/portal.html) placed RG10 and OX948 sequences for the FAD3A gene on the chromosome Gm14 at the locus Glyma.14g194300 (FAD3A) between SSR markers Satt534 and Satt726 in soybean sequence map (position Gm14:45,935,668..45,939,896). The Fan locus and five linolenic acid QTL [Linolen 3-3 (Reinprecht et al., 2006), Linolen 10-1 and Linolen 10-2 (Spencer et al., 2004), Linolen 11-1 and Linolen 11-2 (Xie et al., 2012); SoyBase] were mapped to the same region in the genetic GmComposite_2003 map (Figure 3A).

\section{RG10 × OX948}

In the current work, to fine map the FAD $3 A$ gene, six new SSR markers were selected from the FAD $3 A$ gene region. However, only one marker (Sat_424) was polymorphic between the parents (RG10 and OX948) and its addition to the map did not change position of the FAD $3 A$ gene (Figure 3A). The current size of the chromosome Gm14-based linkage group (B2) in the RG10 $\times$ OX948 population is $81.1 \mathrm{cM}$.

\section{Validation of a Major Linolenic Acid QTL in the PI 361088B x OX948 Population}

To validate the linolenic acid QTL detected with the RG10 $\times$ OX948 population, markers on chromosomes Gm02, Gm11, Gm14, and Gm18 were scored for individuals from the PI $361088 \mathrm{~B} \times$ OX948 population. A relatively small set of SSR markers on four chromosomes was shared between the two populations. In the PI $361088 \mathrm{~B} \times$ OX948 population, chromosome Gm14 (B2) contains eight markers, four of which are shared with the mapping RG10 $\times$ OX948 population. A major QTL (LOD 8), which explains 59\% variability for the linolenic acid content in the PI $361088 \mathrm{~B} \times$ OX948 population was detected between the markers Satt534 and Satt560. This is the same region as the location of the major linolenic acid QTL Linolen 3-3 (Reinprecht et al., 2006; SoyBase) in the RG10 × OX948 mapping population (Figure 3A) and associated RG10 and OX948 FAD3A gene sequences (locus Glyma.14g194300).

\section{FAD3B Gene, Fan3 Allele and Linolenic Acid QTL}

The RG10 and OX948 sequences for the FAD3B gene were placed on the chromosome $\mathrm{Gm} 02$ at the position 


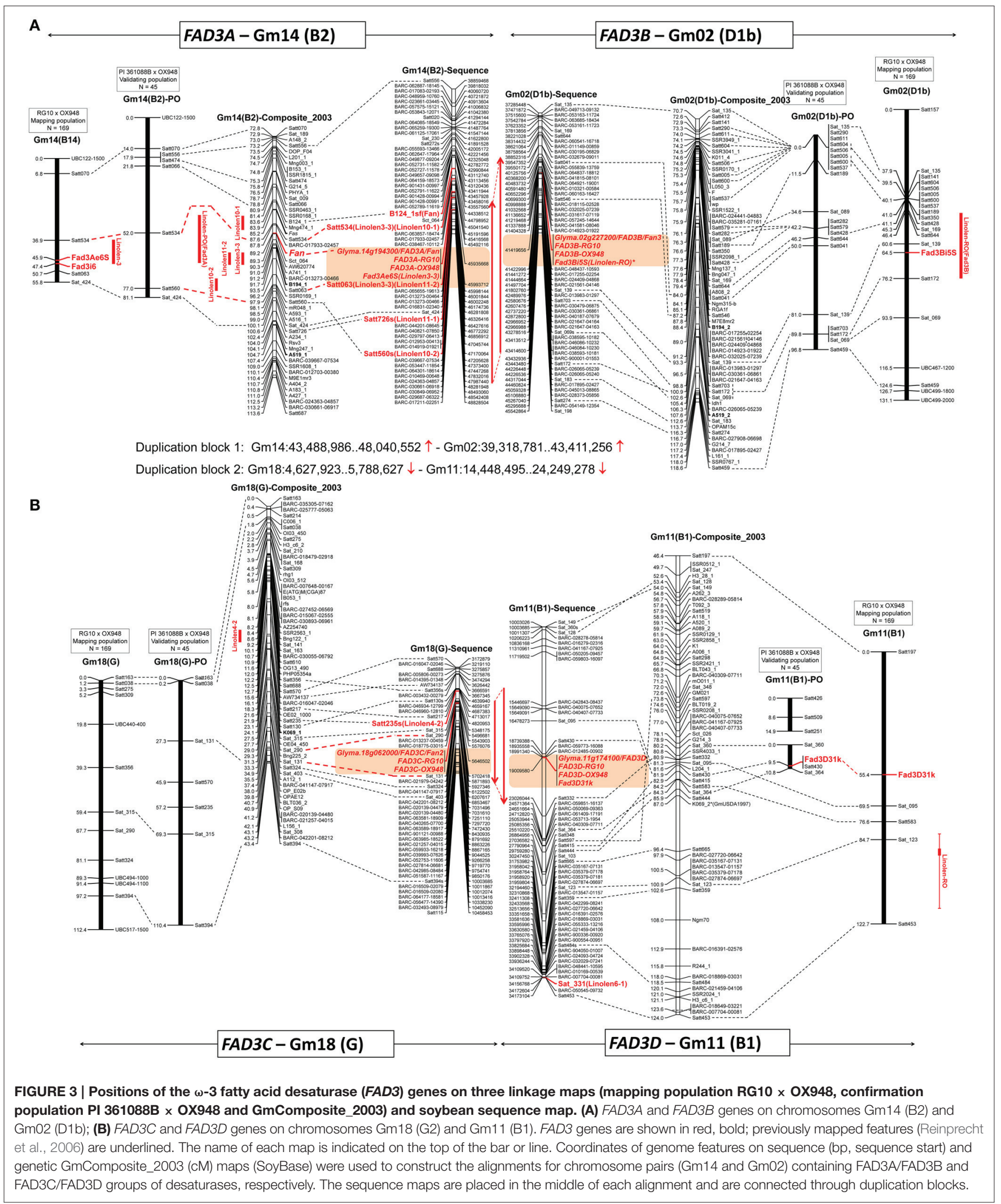




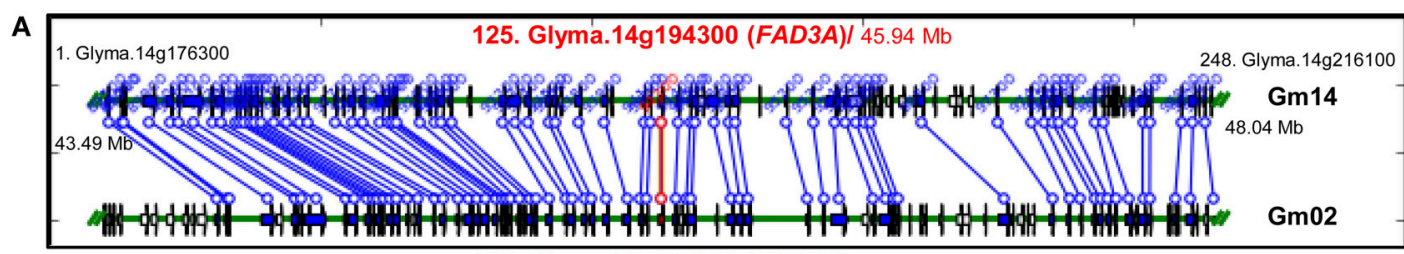

$\begin{array}{lll}\text { 1. Glyma.02g208200/ 39.32 Mb 125. Glyma.02g227200 (FAD3B)/ 41.42 Mb 248. Glyma.02g246000/ 43.41 Mb } & \text { 16 }\end{array}$
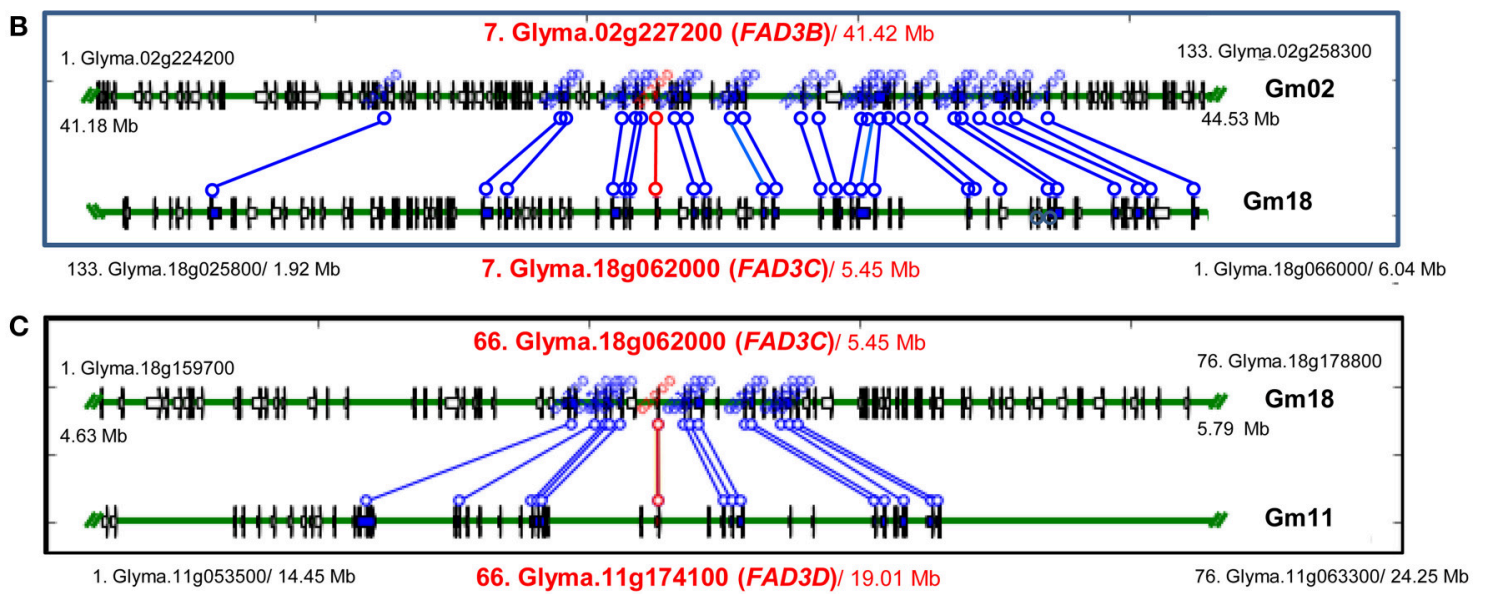

FIGURE 4 | Syntenic regions containing FAD3 genes in soybean genome. (A) Regions containing $F A D 3 A$ and $F A D 3 B$ genes; (B) Regions containing $F A D 3 B$ and $F A D 3 C$ (in reverse orientation) genes; (C) Regions containing FAD3C and FAD3D genes. Syntenic regions were identified using Plant Genome Duplication Database (PGDD). All blocks for the query locus displayed $\pm 500 \mathrm{~Kb}$ region. Query locus, as well as first and last loci in each block are indicated. Each locus identifier consists of a number, indicating locus order in the syntenic block, locus name and a start position in the block (Mb).

Gm02:41,419,656..41,423,881 at the locus Glyma.02g227200 $(F A D 3 B)$ in the soybean sequence map (Wm82.a2.v1). There was no linolenic acid QTL on chromosome Gm02 (D1b) of the GmComposite_2003 genetic map corresponding to the FAD3B gene location in the sequence map (Figure 3A).

\section{RG10 $\times 0 \times 948$}

A SNP marker FAD3B-i5SNP, developed previously for the mutation in the FAD3B gene (Reinprecht et al., 2009), mapped initially to the end of the top portion of the chromosome Gm02 (D1b, 21.4cM from the SSR marker Sat_135) in the RG10 × OX948 map (not shown). To fine map this region, 21 SSR markers were selected in the region of the FAD3B gene and 12 were placed on the RG10 × OX948 map. The addition of new markers allowed fragments of chromosome Gm02 (D1b) to be joined. The current size of this chromosomebased linkage group in the RG10 $\times$ OX948 map is $131.1 \mathrm{cM}$ and it contains 21 markers. The FAD3B gene/marker FAD3B-i5SNP is positioned between the SSR markers Sat_139 $(3.9 \mathrm{cM})$ and Satt172 $(11.7 \mathrm{cM})$ in the region of the newly identified linolenic acid QTL [Linolen-RO(FAD3B), Figure 4A] and corresponds well with the in silico position of the RG10 and OX948 FAD3B gene sequences at the locus Glyma.02g227200 (FAD3B). This QTL (LOD 6.12) explained approximately $10 \%$ variability for linolenic acid content and was consistently expressed in all three Ontario locations (Harrow 8\%, Woodslee 9\%, and Ridgetown
$11 \%)$ with an additive effect ranging from $-9.74 \mathrm{~g} \mathrm{~kg}^{-1}$ (Harrow) to $-11.14 \mathrm{~g} \mathrm{~kg}^{-1}$ (Woodslee).

\section{PI 361088B x OX948}

Chromosome Gm02 (D1b) in the validating population contains 20 markers, 10 of which are in common with the RG10 $\times$ OX948 mapping population. No QTL was identified in the region that corresponds to the region of the minor linolenic acid QTL (markers Sat_139 and Satt172) and the associated FAD3B gene (locus Gm.02g227200) in the RG10 × OX948 mapping population (Figure 3A).

\section{FAD3C Gene, Fan2 Allele, and Linolenic Acid QTL}

The RG10 and OX948 FAD3C gene sequences were mapped to the chromosome Gm18 (G) at the locus Glyma.18g062000 (FAD3C, position Gm18:5,646,502..5,649,337) between SSR markers Satt217 and Satt324 on the soybean sequence map (Wm82.a2.v1). QTL Linolen 4-2 (SoyBase; Panthee et al., 2006) was mapped to the same location on the chromosome Gm18 (G) in the GmComposite_2003 genetic map.

\section{RG10 × OX948}

Nine SSR markers were selected from the FAD3C gene region and two markers (Sat_315 and Sat_290) were placed on the RG10 $\times$ OX948 map in the region predicted to contain the FAD3C gene, between previously mapped markers Satt356 and 
Satt324 (Figure 4B). A SNP marker for the FAD3C gene was developed based on differences in the $5^{\prime}$ UTR region between RG10 and OX948 (Figure S3). Optimization of the marker and subsequent mapping are underway. The size of the chromosome Gm18-based linkage group (G) in the RG10 × OX948 population is $112.4 \mathrm{cM}$. No linolenic acid QTL was detected in the FAD3C gene region of the RG10 $\times$ OX948 map (Figure 3B).

\section{PI 361088B x OX948}

Chromosome Gm18 (G) in the PI 361088B × OX948 derived map contains only seven markers. Four SSR markers were common between the mapping RG10 $\times$ OX948 population and the validating PI $361088 \mathrm{~B} \times$ OX948 population. No markers were mapped and no linolenic acid QTL were detected in the region corresponding to the FAD3C gene (locus Glyma.18g062000) in sequence map (Figure 3B).

\section{FAD3D Gene and Linolenic Acid QTL}

The RG10 and OX948 FAD3D gene sequences were mapped on the chromosome Gm11 (B1) at the locus Glyma.11g174100 (FAD3D, position Gm11:19,009,581..19,012,951) on the soybean sequence map (Wm82.a2.v1). No associations to the Fan alleles were reported and there is no linolenic acid QTL on the chromosome Gm11 (B1) of the GmComposite_2003 genetic map (SoyBase), which corresponds to the FAD3D gene location in soybean sequence map (Figure 3B).

\section{RG10 $\times$ OX948}

Fourteen SSR markers were selected from the FAD $3 D$ gene region and screened for polymorphism with the parental genomic DNA. Three SSR markers were mapped on the chromosome Gm11 (B1) in the RG10 × OX948 population. A SNP marker (Fad3D31k) for the FAD3D gene was developed, based on the difference in the $3^{\prime}$ UTR region in RG10 and OX948 sequences (Figure S4). It mapped to the chromosome Gm11 (B1), $14.1 \mathrm{cM}$ from the SSR marker Sat_095 (Figure 4B). The size of the chromosome Gm11based linkage group (B1) in the RG10 $\times$ OX948 population is $67.3 \mathrm{cM}$. No linolenic acid QTL was detected in the FAD3D gene region of the RG10 × OX948 map. However, a minor QTL associated with the linolenic acid was detected downstream of the $F A D 3 D$ gene-based marker location, which was associated with the marker Sat_123 (Figure 3B).

\section{PI 361088B x OX948}

In the validating population, chromosome Gm11 (B1) is broken into two segments with three and four markers, respectively. The FAD3D gene-based marker (Fad3D31k) mapped to the bottom portion of the chromosome linked to the SSR marker Satt 430 and $8.7 \mathrm{cM}$ apart from Satt298. This position corresponds well to the region of the RG10 and OX948 FAD3D gene sequences (locus Glyma.11g174100) in the RG10 × OX948 mapping population. Moreover, this is the only marker connection between the two populations. However, no QTL was detected in the area of the FAD3D gene in the PI $361088 \mathrm{~B} \times$ OX948 population.

\section{DISCUSSION}

\section{Linolenic Acid Content and $\omega-3$ Fatty Acid Desaturase Activity in Parental Lines}

The low linolenic acid parental genotypes used in the present study, RG10 and PI 361088B, had significantly different levels of linolenic acid and each had significantly lower level of linolenic acid compared to the wild-type parent, OX948. The linolenic acid level for PI 36188B was higher in this study $\left(54 \mathrm{~g} \mathrm{~kg}^{-1}\right)$ than previously reported $\left(39 \mathrm{~g} \mathrm{~kg}^{-1}\right)$ by Rennie and Tanner (1989). A similar difference between studies was reported for the line C1640 (Brummer et al., 1995), and was probably due to testing in different environments.

It was shown previously that reduced levels of linolenic acid in lines RG10 and PI 361088B are controlled by homozygous recessive alleles with additive effects at the Fan locus (Rennie et al., 1988; Stojšin et al., 1998; Reinprecht et al., 2005). This locus encodes $\omega-3$ FAD (Brummer et al., 1995), which catalyzes the desaturation of linoleic to linolenic acid. Wilson et al. (1990) suggested that in Glycine max, values of 18:2 desaturation lower than $9 \%$ are associated with recessive alleles for $\omega-3$ FAD. Conversely, values equal or higher than this threshold value are associated with dominant allele. According to this criteria, the low linolenic acid lines RG10 and PI 361088B are homozygous recessive for $\omega-3 \mathrm{FAD}$. However, the activity of $\omega-3 \mathrm{FAD}$ in line PI 361088B was more than double that observed in line RG10 $(18: 2 \mathrm{D}=3.4$ and $7.8 \%$, respectively). Consistent with this, the level of linolenic acid in RG10 seeds was about half of that in seeds of PI 361088B. In contrast, the high level of linolenic acid in the wild-type parent (OX948) indicates that it contained a pair of dominant alleles for $\omega-3$ FAD. Furthermore, the estimate of $20.5 \%$ for the relative $18: 2$ desaturation value in OX948 was significantly higher than typically found for dominant $\omega-3$ FAD alleles in G. max. This estimate is similar to the relatively high levels of 18:2 desaturation found in Glycine soja plant introductions. Values of $16.8 \%$ in PI 342434 for 18:2 desaturation and $21.1 \%$ in PI 424031 have previously been reported by Pantalone et al. (1997). These authors suggested that "different complement or additional copies of genes" determine levels of linolenic acid in G. soja and this may apply to the high levels of linolenic acid detected in the line OX948 in the present study.

\section{Sequences of FAD3 Genes in RG10 and OX948}

The whole length genomic DNA sequences we obtained for $F A D 3 A, F A D 3 B, F A D 3 C$, and FAD3D genes in RG10 and OX948 confirmed that the microsomal $\omega-3$ FADs in soybean are 78 to 95\% similar (ORF). All four FAD3 genes have a similar structure and contain eight exons, as reported previously (Iba et al., 1993). In general, the sizes of the exons were well conserved among the FAD3 genes and corresponded well to the sizes of FAD3 exons in Arabidopsis. The most variable was exon 1, which has different sizes in all four FAD3 genes. Exon 8 was slightly shorter in FAD3A and FAD3B (183 bp) compared to the FAD3C and $F A D 3 D$ (192 bp). However, compared to exons, intron sizes were more variable. All seven introns have different sizes in the four FAD3 genes. Based on the sequence similarity and exon/intron 
structure, the four FAD3 genes can be separated into two groups consisting of $F A D 3 A / F A D 3 B$ and $F A D 3 C / F A D 3 D$, respectively. Within each group, the members share $95 \%$ sequence similarity (coding region) and they have similarly sized introns. The longer introns in the $F A D 3 A / F A D 3 B$ gene group are 2, 6, and 7, while the longest introns in the FAD $3 C / F A D 3 D$ group are 3 and 4 . There is 78 to $80 \%$ sequence similarity between the groups. The FAD $3 A$ ORF encodes the smallest protein while the ORFs of other three $F A D 3$ genes encode slightly larger proteins of similar sizes (except for the FAD $3 A$ and $F A D 3 B$ in mutant RG10; Figure S5).

\section{In silico Map of FAD3 Genes in RG10 and OX948}

Previously, we mapped $F A D 3 A$ gene to the linkage group B2 (Gm14) in the RG10 × OX948 population (Reinprecht et al., 2006). The position of the FAD $3 A$ gene was confirmed by in silico mapping of the RG10 and OX948 sequences. BLAST searches against soybean genome (Wm82.a2.v1) placed RG10 and OX948 sequences for the FAD3A gene on the chromosome Gm14 at the locus Glyma.14g194300 (FAD3A, position $\mathrm{Gm} 14: 45,935,668 . .45,939,896)$, the $F A D 3 B$ gene on the chromosome Gm02 at the locus Glyma.02g227200 (FAD3B, position $\mathrm{Gm} 02: 41,419,656 ; ; 41,423,881)$, the $F A D 3 C$ gene on the chromosome Gm18 at the locus Glyma.18g062000 (FAD3C, position $\mathrm{Gm} 18: 5,646,502 . .5,649,337)$ and the $F A D 3 D$ gene on the chromosome Gm11 at the locus Glyma.11g174100 (FAD3D, position Gm:19,009,581..19,012,951) (Figure 3). The positions of the FAD3 genes in the soybean sequence map (Wm82.a2.v1) are slightly different from those reported in an earlier version of the soybean genome sequence (Chi et al., 2011) and might change again with the additional genome annotations.

The availability of the complete genome sequences for numerous plant species allows the organization of the individual genomes to be studied, as well as enables comparison of the genomes at the nucleotide level. Synteny analysis performed with soybean FAD3 genes against complete genome sequences available for 47 flowering plant species in PGDD identified numerous syntenic regions. Syntenic blocks were of various sizes and contained 6 to 302 gene anchors. The multiple copies of genes in soybean arise from its tetraploid nature (Shoemaker et al., 1996; Schlueter et al., 2007; Roulin et al., 2013). Thus, four copies of $\omega-3$ FADs are theoretically expected and in fact, two pairs of homologous $\omega-3$ FAD regions were detected in the soybean genome sequence (Wm82.a2.v) and, by in silico mapping. These were located on chromosomes Gm14 (B2), Gm02 (D1b), Gm18 (G), and Gm11 (B1) (Chi et al., 2011; SoyBase). The regions on chromosomes Gm14 (B2, FAD3A) and $\mathrm{Gm} 02$ (D1b, FAD3B) are syntenic, as well as regions on chromosomes Gm18 (G, FAD3C) and Gm11 (B1, FAD3D).

\section{Position of the FAD3 Genes in the Linolenic Acid QTL Regions of the RG10 × OX948 Linkage Map}

Five desaturase gene-specific markers (FAD3i1, FAD3i3, FAD3T, FAD3M, and FAD3B) designed earlier based on the L22964 sequence (Yadav et al., 1993), were removed from the RG10 ×
OX948 map (Reinprecht et al., 2006). The addition of new SSR and FAD3 gene-specific markers developed in this study to the chromosome Gm14 (B2), Gm02 (D1b), and Gm11 (B1) changed some distances compared to the original RG10 $\times$ OX948 map. It also helped to join two segments of chromosome Gm11 (B1), which initially contained only two SSR markers (Sat_123 and Satt453), as well as two segments of chromosome Gm02 (D1b) in the RG10 × OX948 map.

\section{FAD3A and FAD3B Genes}

The addition of a single SSR marker to the chromosome Gm14 (B2) did not change the position of the previously mapped FAD3A gene (Reinprecht et al., 2009). This gene was mapped as a FAD3A gene-based marker to the region of a major linolenic acid QTL (Linolen 3-3) on the chromosome Gm14 (B2) in the RG10 × OX948 map (Reinprecht et al., 2006; SoyBase), which is the same genomic region as the previously mapped fan allele that conditions low linolenic acid content in C1640 (Brummer et al., 1995). This is also consistent with the previous placement of the microsomal $\omega-3$ FAD gene on the chromosome Gm14 (B2) (Byrum et al., 1995) and corresponds well with the in silico position of the RG10 and OX948 FAD3A gene sequences (this study).

The FAD $3 B$ gene-based marker developed previously (Reinprecht et al., 2009) was mapped on the chromosome Gm02 (D1b) of the RG10 × OX948 map, between SSR markers Sat_139 and Satt172, in the region of the newly detected linolenic acid QTL [Linolen-RO (FAD3B)]. This corresponds well with the in silico position of the RG10 and OX948 FAD3B gene sequences.

\section{FAD3C and FAD3D Genes}

Because of the problem with amplification, optimization of a SNP marker developed for the FAD3C gene (difference in the 5'UTR region in RG10 and OX948 sequences) and subsequent mapping are underway. No linolenic acid QTL was detected in the FAD $3 C$ gene region with (based on in silico map) the RG10 $\times$ OX948 derived RILs. The FAD3D gene-based marker mapped to the chromosome Gm11 (B1), between SSR markers Sat_095 and Satt197, which corresponds well to the RG10 and OX948 FAD3D gene sequence position in the soybean in silico map. No linolenic acid QTL was detected in the FAD3D gene region of the RG10 $\times$ OX948 derived map. However, a minor QTL that was associated with the marker Sat_123, was detected downstream of this region.

\section{Validation of a Major Linolenic Acid QTL (Linolen 3-3) in the PI 361088B x OX948 Population}

In soybean, significant work was done on mapping genomic regions associated with the seed linolenic acid content. Currently, 44 linolenic acid QTL (identified in 10 independent studies) have been deposited in SoyBase (23 Sep 2015). However, information for several QTL from these studies are unavailable since the markers associated with them were not present on the consensus and/or composite genetic maps. Xie et al. (2012) developed several FAD3 gene-based markers and mapped six linolenic acid QTL in a $\mathrm{F}_{5: 7}$ population derived from the cross $\mathrm{He}$ Feng $25 \times$ Dongnong L-5 over several environments. However, 
QTL Linolen 11-3 (marker FAD3b-1) and Linolen 11-4 (marker FAD3c-2) were not reported in SoyBase since these markers are not present on the GmComposite_2003 map. In addition, QTL Linolen 11-2 was associated with SSR marker Satt063 (SoyBase) but not FAD3a-4 marker that was reported originally by Xie et al. (2012). In the current study, sequences of these markers were BLASTed against soybean genome, but because of absence of any significant hits they were not placed on the sequence map. There are two additional linolenic acid QTL studies not cataloged in SoyBase. Brummer et al. (1995) mapped the Fan locus between RFLP markers B124_1 and B194_1 on linkage group B2 (Gm14) in an $\mathrm{F}_{2}$ population developed from the $G$. $\max (\mathrm{C} 1694) \times G$. soja (PI 479750) cross. The QTL at this position explained most of the variation (85\%) for the linolenic acid content in that population. Recently, Akond et al. (2014) identified six QTL associated with the levels of linolenic acid in an $\mathrm{F}_{5: 7}$ population developed from the MD96-5722 $\times$ Spencer cross. These QTL were mapped on four chromosomes (Gm13, Gm14, Gm15, and Gm16) and explained 5 to $23 \%$ variability for the linolenic acid content in this population. However, linolenic acid QTL reported in both studies as well as three linolenic acid QTL (Linolen 8-1, Linolen 8-2, and Linolen 8-3; SoyBase) reported by Kim et al. (2010) should be considered as putative QTL since their identification was based on a single environment data.

Linolenic acid QTL deposited in SoyBase were identified using diverse populations (type and size), which were evaluated in different environments and analyzed with various methods. Using a meta analysis of publicly available QTL information, $\mathrm{Hu}$ et al. (2011) integrated major QTL for numerous important agronomic traits including fatty acids. They identified three regions containing linolenic acid QTL: three overlapping linolenic acid QTL on chromosome Gm14 (B2) between markers Sat_230 and Satt560, a single QTL on chromosome Gm18 (G) between markers Satt356 and Sat_290 and two regions each with two overlapping QTL on chromosome Gm15 (E). However, to be useful for plant breeding published QTL need to be confirmed in an independent population. QTL confirmation will provide new insight into the strengths as well as limitations of QTL implementation in MAS (Young, 1999).

Although numerous QTL for different traits were identified in soybean, very few of them were verified in independent populations. For example, QTL for soybean cyst nematode resistance, initially identified in an $\mathrm{F}_{2: 3}$ population, were confirmed in $\mathrm{F}_{6: 7}$ RILs developed from the same Magellan (susceptible) $\times$ PI 438489B (resistant) cross (Vuong et al., 2011), while QTL for seed weight, protein and oil contents were confirmed in different genetic backgrounds by two research groups (Fasoula et al., 2004; Pathan et al., 2013). Seven QTL for linolenic acid were mapped on the chromosome Gm14

\section{REFERENCES}

Akond, M., Liu, S., Boney, M., Kantartzi, S. K., Meksem, K., Bellaloui, N., et al. (2014). Identification of quantitative trait loci (QTL) underlying protein, oil, and five major fatty acids' contents in soybean. Am. J. Plant Sci. 5, 158-167. doi: 10.4236/ajps.2014.51021
(B2) in five different populations by four research groups (SoyBase). Four of these QTL (Linolen 10-1, Linolen 10-2, Linolem 11-2) were identified in the region $(86.3-98.9 \mathrm{cM}$, GmComposite_2003) of the Fan locus. The major linolenic acid QTL (Linolen 3-3) was detected in all three testing locations (Harrow, Ridgetown, and Woodslee, ON) and explained very similar amounts of the total phenotypic variability $\left(R^{2}=0.76\right)$ for linolenic acid content in the RG10 × OX948 mapping population (Reinprecht et al., 2006). This is an example of the most important type of QTL for breeders because it was associated with the FAD3A gene (Reinprecht et al., 2009) and confirmed in the PI $361088 \mathrm{~B} \times$ OX948 population $\left(R^{2}=0.59\right)$, which may lead to the stability in breeding for low linolenic acid trait. However, because of the small size of the PI $361088 \mathrm{~B} \times$ OX948 confirmation population $(N=45)$ additional work is required to verify that. In this study, we identified a new QTL associated with linolenic acid content in the RG10 × OX948 population. It mapped to the chromosome Gm02 (D1b), which explained $\sim 10 \%$ variation for the trait and was associated with the $F A D 3 B$ gene. The usefulness of the previously developed $F A D 3 A$ and $F A D 3 B$ gene mutation-based markers was confirmed with a number of RG10-derived low linolenic acid lines and cultivars with normal level of linolenic acid (Reinprecht et al., 2009). In the current work, we sequenced all four microsomal $F A D 3$ genes and identified numerous variations between parental lines of mapping population (RG10 and OX948) as well as reference Wm82. In the future, these polymorphisms could be used to develop additional FAD3 gene-based markers. To be useful for the soybean breeders globally, these markers need to be validated in diverse germplasm.

\section{AUTHOR CONTRIBUTIONS}

All authors listed, have made substantial, direct, and intellectual contribution to the work, and approved it for publication.

\section{ACKNOWLEDGMENTS}

Authors acknowledge funding by the Ontario Soybean Growers' and Hannam Soybean Utilization Fund. Technical assistance by Julia Zilka, Jan Brazolot, and UofG soybean crew is greatly appreciated.

\section{SUPPLEMENTARY MATERIAL}

The Supplementary Material for this article can be found online at: http://journal.frontiersin.org/article/10.3389/fgene. 2016.00038
Anai, T., Yamada, T., Kinoshita, T., Rahman, S. M., and Takagi, Y. (2005). Identification of corresponding genes for three low $\alpha$-linolenic acid mutants and elucidation of their contribution to fatty acid biosynthesis in soybean seed. Plant Sci. 168, 1615-1623. doi: 10.1016/j.plantsci.2005.02.016

Bannon, C. D., Craske, J. D., Felder, D. L., Garland, I. J., and Norman, L. M. (1987). Analysis of fatty acid methyl esters with accuracy and reliability. VI. Rapid 
analysis by split injection capillary gas liquid chromatography. J. Chromatogr. 407, 231-241. doi: 10.1016/S0021-9673(01)92621-4

Bilyeu, K. D., Gillman, J. D., and LeRoy, A. R. (2011). Novel FAD3 mutant allele combinations produce soybeans containing $1 \%$ linolenic acid in the seed oil. Crop Sci. 51, 259-264. doi: 10.2135/cropsci2010.01.0044

Bilyeu, K. D., Palavalli, L., Sleper, D. D., and Beuselinck, P. R. (2003). Three microsomal omega-3 fatty-acid desaturase genes contribute to soybean linolenic acid levels. Crop Sci. 43, 1833-1838. doi: 10.2135/cropsci2003.1833

Bilyeu, K., Palavalli, L., Sleper, D. A., and Beuselinck, P. R. (2006). Molecular genetic resources for development of $1 \%$ linolenic acid soybean. Crop Sci. 46, 1913-1918. doi: 10.2135/cropsci2005.11-0426

Bilyeu, K., Palavalli, L., Sleper, D., and Beuselinck, P. R. (2005). Mutations in soybean microsomal omega-3 fatty acid desaturase genes reduce linolenic acid concentration in soybean seeds. Crop Sci. 45, 1830-1836. doi: $10.2135 /$ cropsci2004.0632

Browse, J., and Somerville, C. (1991). Glycerolipid synthesis: biochemistry and regulation. Annu. Rev. Plant Physiol. Plant Mol. Biol. 42, 467-506. doi: 10.1146/annurev.pp.42.060191.002343

Browse, J., Warwick, N., Somerville, C. R., and Slack, C. R. (1986). Fluxes through the prokaryotic and eukaryotic pathways of lipid synthesis in the '16:3' plant Arabidopsis thaliana. Biochem. J. 235, 25-31. doi: 10.1042/bj2350025

Brummer, E. C., Nickell, A. D., Wilcox, J. R., and Shoemaker, R. C. (1995). Mapping the Fan locus controlling linolenic acid content in soybean oil. J. Hered. 86, 245-247.

Byrum, J. R., Kinney, A. J., Shoemaker, R. C., and Diers, B. W. (1995). Mapping of the microsomal and plastid omega-3 fatty acid desaturases in soybean [Glycine $\max (\mathrm{L}$.$) Merr.]. Soybean Genet. Newslett. 22, 181-184.$

Chappell, A. S., and Bilyeu, K. D. (2006). A GmFAD3A mutation in the low linolenic acid soybean mutant C1640. Plant Breed. 125, 535-536. doi: 10.1111/j.1439-0523.2006.01271.x

Chappell, A. S., and Bilyeu, K. D. (2007). The low linolenic acid soybean line PI 361088B contains a novel GmFAD3A mutation. Crop Sci. 47, 1705-1710. doi: 10.2135/cropsci2006.12.0783

Chenna, R., Sugawara, H., Koike, T., Lopez, R., Gibson, T. J., Higgins, D. G., et al. (2003). Multiple sequence alignment with the Clustal series of programs. Nucleic Acids Res. 31, 3497-3500. doi: 10.1093/nar/gkg500

Cherif, A., Dubacq, J. P., Mache, A., Oursel, R., and Tremolieres, A. (1975). Biosynthesis of $\alpha$-linolenic acid by desaturation of oleic and linoleic acids in several organs of higher and lower plants and in algae. Phytochemistry 14, 703-706. doi: 10.1016/0031-9422(75)83018-4

Chi, X., Yang, Q., Lu, Y., Wang, J., Zhang, Q., Pan, L., et al. (2011). Genome-wide analysis of fatty acid desaturases in soybean (Glycine max). Plant Mol. Biol. Rep. 29, 769-783. doi: 10.1007/s11105-010-0284-z

Clemente, T. E., and Cahoon, E. B. (2009). Soybean oil: genetic approaches for modification of functionality and total content. Plant Physiol. 151, 1030-1040. doi: $10.1104 /$ pp.109.146282

Doyle, J. J., and Doyle, J. L. (1990). Isolation of plant DNA from fresh tissue. Focus $12,13-15$.

Fasoula, V. A., Harris, D. K., and Boerma, H. R. (2004). Validation and designation of quantitative trait loci for seed protein, seed oil and seed weight from two soybean population. Crop Sci. 44, 1218-1225. doi: 10.2135/cropsci2004.1218

Gilman, J. D., and Bilyeu, K. D. (2012). "Gene and alleles for quality traits on the soybean genetic/physical map," in Designing Soybeans for 21st Century Markets, ed R. F. Wilson (Urbana, IL: AOCS Press), 67-96.

Goodstein, D. M., Shu, S., Howson, R., Neupane, R., Hayes, R. D., Fazo, J., et al. (2012). Phytozome: a comparative platform for green plant genomics. Nucleic Acids Res. 40(Database issue), D1178-D1186. doi: 10.1093/nar/gkr944

Grant, D., Nelson, R. T., Cannon, S. B., and Shoemaker, R. C. (2010). SoyBase, the USDA-ARS soybean genetics and genomics database. Nucleic Acids Res. 38, D843-D846. doi: 10.1093/nar/gkp798

Guerra, D. J., and Holbrook, L. (1988). "Plant fatty acid synthesis: sites of metabolic regulation and potential for genetic engineering," in Proceedings - World Conference on Biotechnology for the Fats and Oils Industry, ed T. H. Applewhite (Champaign, IL: The American Oil Chemist's Society), 39-42.

Hammond, E. G., and Fehr, W. R. (1975). Oil quality improvement in soybeans, Glycine max (L.) Merr. Fette Seifen Anstrichmittel 77, 97-101. doi: 10.1002/lipi.19750770304

Harwood, J. L. (1988). Fatty acid metabolism. Annu. Rev. Plant Physiol. Plant Mol. Biol. 39, 101-138. doi: 10.1146/annurev.pp.39.060188.000533
Hu, G., Chen, Q., Liu, C., Jiang, H., Wang, J., and Qi, Z. (2011). "Integration of major QTLs of important agronomic traits in soybean," in Soybean-Molecular Aspects of Breeding, ed A. Sudaric (Rijeka: InTech open access publisher, www.intechopen.com), 81-118.

Iba, K., Gibson, S., Nishiuchi, T., Fuse, T., Nishimura, M., Arondel, V., et al. (1993). A gene encoding a chloroplast $\omega-3$ fatty acid desaturase complements alterations in fatty acid desaturation and chloroplast copy number of the FAD7 mutant of Arabidopsis thaliana. J. Biol. Chem. 268, 24099-24105.

Khanna, K. R., and Singh, S. P. (1991). "Genetics of fatty acid composition and breeding for modified seed oils," in Biochemical Aspects of Crop Improvement, ed K. R. Khanna (Boca Raton, FL: CRC Press), 255-281.

Kim, H.-K., Kim, Y.-C., Kim, S.-T., Son, B.-G., Choi, Y.-W., Kang, J.-S., et al. (2010). Analysis of quantitative trait loci (QTLs) for seed size and fatty acid composition using recombinant inbred lines in soybean. J. Life Sci. 20, 1186-1192. doi: 10.5352/JLS.2010.20.8.1186

Kinney, A. J. (1997). "Development of genetically engineered oilseeds," in Physiology, Biochemistry and Molecular Biology of Plant Lipids, eds J. P. Williams, M. Uddin Khan, and N. Wan Lem (Dordrecht; Boston, MA; London: Kluwer Academic Publishers), 298-300.

Kosambi, D. D. (1944). The estimation of map distances from recombination values. Ann. EuGenet. 12, 172-175. doi: 10.1111/j.1469-1809.1943.tb02321.x

Lander, E. S., Green, P., Abrahamson, J., Barlow, A., Daly, M. J., Lincoln, S. E., et al. (1987). MAPMAKER: an interactive computer package for constructing primary linkage maps of experimental and natural populations. Genomics 1, 174-181. doi: 10.1016/0888-7543(87)90010-3

Lee, J.-D., Bilyeu, K. D., and Shannon, J. G. (2007). Genetics and breeding for modified fatty acid profile in soybean seed oil. J. Crop Sci. Biotech. 10, 201-210.

Lee, T. H., Tang, H., Wang, X., and Paterson, A. H. (2013). PGDD: a database of gene and genome duplication in plants. Nucleic Acids Res. 41, D1152-D1158. doi: $10.1093 / \mathrm{nar} / \mathrm{gks} 1104$

Lyons, E., and Freeling, M. (2008). How to usefully compare homologous plant genes and chromosomes as DNA sequences. Plant J. 53, 661-673. doi: 10.1111/j.1365-313X.2007.03326.X

Lyons, E., Pedersen, B., Kane, J., Alam, M., Ming, R., Tang, H., et al. (2008). Finding and comparing syntenic regions among Arabidopsis and the outgroups papaya, poplar and grape: CoGe with rosids. Plant Physiol. 148, 1772-1781. doi: $10.1104 /$ pp.108.124867

Mounts, T. L., Warner, K., List, G. R., Kleiman, R., Fehr, W. R., Hammond, E. G., et al. (1986). Effect of altered fatty acid composition on soybean oil stability. J. Am. Oil Chem. Soc. 65, 624-628. doi: 10.1007/BF02540691

Nishiuchi, T., Nishimura, M., Arondel, V., and Iba, K. (1994). Genomic nucleotide sequence of a gene encoding a microsomal omega- 3 fatty acid desaturase from Arabidopsis thaliana. Plant Physiol. 105, 767-768. doi: 10.1104/pp.105.2.767

Ohlrogge, J. B., Jaworski, J. G., and Post-Beittenmiller, D. (1993). "De novo fatty acid biosynthesis," in Lipid Metabolism in Plants, ed T.S. Moore, Jr. (Boca Raton, FL: CRC Press), 3-32.

Pantalone, V. R., Rebetzke, G. J., Burton, J. W., and Wilson, R. F. (1997). Genetic regulation of linolenic acid concentration in wild soybean Glycine soja accessions. J. Am. Oil Chem. Soc. 74, 159-163. doi: 10.1007/s11746-997-0162-5

Pathan, S. M., Vuong, T., Clark, K., Lee, J.-D., Shannon, J. G., Roberts, C. A., et al. (2013). Genetic mapping and confirmation of quantitative trait loci for seed protein and oil contents and seed weight in soybean. Crop. Sci. 53, 765-774. doi: $10.2135 /$ cropsci2012.03.0153

Panthee, D. R., Pantalone, V. R., and Saxton, A. M. (2006). Modifier QTL for fatty acid composition in soybean oil. Euphytica 152, 67-73. doi: 10.1007/s10681006-9179-3

Rajcan, I., Hou, G., and Weir, A. (2005). Advances in breeding of seed-quality traits in soybean. J. Crop Improv. 14, 145-174. doi: 10.1300/J411v14n01_07

Reinprecht, Y., Luk-Labey, S., Larsen, J., Poysa, V. W., Yu, K., Rajcan, I., et al. (2009). Molecular basis of the low linolenic acid trait in soybean EMS mutant line RG10. Plant Breed. 128, 253-258. doi: 10.1111/j.1439-0523.2008.01571.x

Reinprecht, Y., Luk-Labey, S.-Y., and Pauls, K. P. (2011). “A versatile soybean recombinant inbred population segregating for low linolenic acid and lipoxygenase nulls - molecular characterization and utility for soymilk and bioproduct production," in Soybean: Molecular Aspects of Breeding, ed A. Sudaric (Rijeka: InTech, www.intechopen.com), 119-146. doi: 10.5772/ 15571

Reinprecht, Y., Poysa, V. W., Yu, K., Rajcan, I., Ablett, G. R., and Pauls, K. P. (2006). Seed and agronomic QTL in low linolenic acid, lipoxygenase-free 
soybean (Glycine $\max$ (L.) Merrill) germplasm. Genome 49, 1510-1527. doi: $10.1139 / \mathrm{g} 06-112$

Reinprecht, Y., Rajcan, I., Poysa, V. W., Ablett, G. R., and Pauls, K. P. (2005). Relationships and inheritance of linolenic acid and seed lipoxygenases in soybean crosses designed to combine these traits. Can. J. Plant Sci. 85, 593-602. doi: 10.4141/P04-098

Rennie, B. D., and Tanner, J. W. (1989). Comparison of the fan alleles in C1640 and the lines A4, PI 123440, and PI 361088B. Soybean Genet. Newslett. 16, 23-25.

Rennie, B. D., Zilka, J., Cramer, M. M., and Beversdorf, W. D. (1988). Genetic analysis of low linolenic acid levels in the soybean line PI 361088B. Crop Sci. 28, 655-657. doi: 10.2135/cropsci1988.0011183X002800040019x

Roulin, A., Auer, P. L., Libault, M., Schlueter, J., Farmer, A., May, G., et al. (2013). The fate of duplicated genes in a polyploid plant genome. Plant J. 73, 143-153. doi: $10.1111 /$ tpj.12026

Rozen, S., and Skaletsky, H. J. (2000). "Primer3 on the WWW for general users and for biologist programmers," in Bioinformatics Methods and Protocols: Methods in Molecular Biology, eds S. Krawetz and S. Misener (Totowa, NJ: Humana Press), 365-386.

Salamov, A. A., and Solovyev, V. V. (2000). Ab initio gene finding in Drosophila genomic DNA. Genome Res. 10, 516-522. doi: 10.1101/gr.10.4.516

SAS Institute Inc (2009). SAS/STAT ${ }^{\circledR} 9.2$ User's Guide. Cary, NC: SAS Institute Inc.

Schlueter, J. A., Lin, J.-Y., Schlueter, S. D., Vasylenko-Sanders, I. F., Deshpande, S., Yi, J., et al. (2007). Gene duplication and paleopolyploidy in soybean and the implications for whole genome sequencing. BMC Genomics 8:330. doi: 10.1186/1471-2164-8-330

Schmutz, J., Cannon, S. B., Schlueter, J., Ma, J., Mitros, T., Nelson, W., et al. (2010). Genome sequence of the palaeopolyploid soybean. Nature 463, 178-183. doi: 10.1038 /nature08670

Shanklin, J., and Cahoon, E. B. (1998). Desaturation and related modifications of fatty acids. Annu. Rev. Plant Physiol. Plant Mol. Biol. 49, 611-641. doi: 10.1146/annurev.arplant.49.1.611

Shanklin, J., Whittle, E., and Fox, B. G. (1994). Eight histidine residues are catalytically essential in a membrane-associated iron enzyme, stearoyl-CoA desaturase, and are conserved in alkane hydroxylase and xylene monooxygenase. Biochemistry 33, 12787-12794. doi: 10.1021/ bi00209a009

Shoemaker, R. C., Polzin, K., Labate, J., Specht, J., Brummer, E. C., Olson, T., et al. (1996). Genome duplication in soybean (Glycine subgenus soja). Genetics 144, 329-338.

Somerville, C. R., and Browse, J. (1991). Plant lipids: metabolism, mutants and membranes. Science 252, 80-87. doi: 10.1126/science.252.5002.80

Spencer, M. M., Landau-Ellis, D., Meyer, E. J., and Pantalone, V. R. (2004). Molecular markers associated with linolenic acid content in soybean. J. Am. Oil Chem. Soc. 81, 559-562. doi: 10.1007/s11746-006-0941-4

Stojšin, D., Luzzi, B. M., Ablett, G. R., and Tanner, J. W. (1998). Inheritance of low linolenic acid level in the soybean line RG10. Crop Sci. 38, 1441-1444. doi: 10.2135/cropsci1998.0011183X003800060006x

Voorrips, R. E. (2002). MapChart: Software for the graphical presentation of linkage maps and QTL. J. Hered. 93, 77-78. doi: 10.1093/jhered/93.1.77
Vuong, T. D., Sleper, D. A., Shannon, J. G., Wu, X., and Nguyen, H. T. (2011). Confirmation of quantitative trait loci for resistance to multiple-HG types of soybean cyst nematode (Heterodera glycines Ichinohe). Euphytica 181, 101-113. doi: 10.1007/s10681-011-0430-1

Wang, S., Basten, C. J., and Zeng, Z.-B. (2011). Windows QTL Cartographer 2.5. Department of Statistics, North Carolina State University, Raleigh, NC. Available online at: http://statgen.ncsu.edu/qtlcart/WQTLCart.htm.

Wilcox, J. R. (1989). "Soybean protein and oil quality," in Proceedings of World Soybean Research Conference, ed A. J. Pascale (Buenos Aires), 28-39.

Wilcox, J. R., and Cavins, J. F. (1985). Inheritance of low linolenic acid content of the seed oil of a mutant in Glycine max. Theor. Appl. Genet. 71, 74-78. doi: $10.1007 / \mathrm{BF} 00278257$

Wilson, R. F., Burton, J. W., and Brim, C. A. (1981). Progress in the selection for altered fatty acid composition in soybeans. Crop Sci. 21, 788-791. doi: 10.2135/cropsci1981.0011183X002100050039x

Wilson, R. F., Burton, J. W., and Kwanyuen, P. (1990). "Effect of genetic modification of fatty acid composition of soybeans on oil quality," in Edible Fats and Oils Processing: Basic Principles and Modern Practices: World Conference Proceedings, ed D. R. Erickson (Champaign, IL: American Oil Chemists' Society), 355-359.

Wilson, R. F. (1991). "Advances in the genetic alteration of soybean oil composition," in Designing value-added soybeans for markets of the future, ed R. F. Wilson (Champaign, IL: American Oil Chemists' Society), 38-52.

Xiaoqiu, H., and Madan, A. (1999). CAP3: a DNA sequence assembly program. Genome Res. 9, 868-877. doi: 10.1101/gr.9.9.868

Xie, D., Han, Y., Zeng, Y., Chang, W., Teng, W., and Li, W. (2012). SSR- and SNP-related QTL underlying linolenic acid and other fatty acids contents in soybean seeds across multiple environments. Mol. Breed. 30, 169-179. doi: 10.1007/s11032-011-9607-5

Yadav, N. S., Wierbicki, A., Aegerter, M., Caster, C. S., Pérez-Grau, L., Kinney, A. J., et al. (1993). Cloning of higher plant $\omega-3$ fatty acid desaturases. Plant Physiol. 103, 467-476. doi: 10.1104/pp.103.2.467

Young, N. D. (1999). A cautiously optimistic vision for marker-assisted breeding. Mol. Breed. 5, 505-510. doi: 10.1023/A:1009684409326

Conflict of Interest Statement: The authors declare that the research was conducted in the absence of any commercial or financial relationships that could be construed as a potential conflict of interest.

The reviewer VS and Handling Editor declared their shared affiliation, and the Handling Editor states that the process nevertheless met the standards of a fair and objective review.

Copyright $\odot 2016$ Reinprecht and Pauls. This is an open-access article distributed under the terms of the Creative Commons Attribution License (CC BY). The use, distribution or reproduction in other forums is permitted, provided the original author(s) or licensor are credited and that the original publication in this journal is cited, in accordance with accepted academic practice. No use, distribution or reproduction is permitted which does not comply with these terms. 